\title{
UCRL-BOOK-206476
}

LAW RENCE LIVERMORE N A TIO N A L LABORATORY

A multi-scale approach to molecular dynamics simulations of shock waves

E. J. Reed, L. E. Fried, M. R. Manaa, J. D. Joannopoulos

September 9, 2004

Chemistry at Extreme Conditions 
This document was prepared as an account of work sponsored by an agency of the United States Government. Neither the United States Government nor the University of California nor any of their employees, makes any warranty, express or implied, or assumes any legal liability or responsibility for the accuracy, completeness, or usefulness of any information, apparatus, product, or process disclosed, or represents that its use would not infringe privately owned rights. Reference herein to any specific commercial product, process, or service by trade name, trademark, manufacturer, or otherwise, does not necessarily constitute or imply its endorsement, recommendation, or favoring by the United States Government or the University of California. The views and opinions of authors expressed herein do not necessarily state or reflect those of the United States Government or the University of California, and shall not be used for advertising or product endorsement purposes. 


\title{
Chapter 10
}

\section{A Multi-Scale Approach to Molecular Dynamics Simulations of Shock Waves}

\author{
Evan J. Reed ${ }^{1}$, Laurence E. Fried ${ }^{2}$, M. Riad $\operatorname{Manaa}^{2}$, J. D. Joannopoulos ${ }^{1}$ \\ ${ }^{1}$ Massachusetts Institute of Technology, Cambridge, MA 02139 \\ ${ }^{2}$ Lawrence Livermore National Laboratory, Livermore, CA 94550
}

\section{INTRODUCTION}

Study of the propagation of shock waves in condensed matter has led to new discoveries ranging from new metastable states of carbon [1] to the metallic conductivity of hydrogen in Jupiter, [2] but progress in understanding the microscopic details of shocked materials has been extremely difficult. Complications can include the unexpected formation of metastable states of matter that determine the structure, instabilities, and time-evolution of the shock wave. [1,3] The formation of these metastable states can depend on the time-dependent thermodynamic pathway that the material follows behind the shock front. Furthermore, the states of matter observed in the shock wave can depend on the timescale on which observation is made. [4,1] Significant progress in understanding these microscopic details has been made through molecular dynamics simulations using the popular non-equilibrium molecular dynamics (NEMD) approach to atomistic simulation of shock compression. [5]

The NEMD method involves creating a shock at one edge of a large system by assigning some atoms at the edge a fixed velocity. The shock propagates across the computational cell to the opposite side. The computational work required by NEMD scales at least quadratically in the evolution time because larger systems are needed for longer simulations to prevent the shock wave from reflecting from the edge of the computational cell and propagating back into the cell. When quantum mechanical methods with poor scaling of computational effort with system size are employed, this approach to shock simulations rapidly becomes impossible. For example, the computational work required for the simulation of a shock is of quadratic order in the simulation duration $\left(O\left(t_{\text {simulation }}^{4}\right)\right)$ for a tight-binding method of force evaluation requiring computational work that scales with the number of atoms $N$ like $O\left(N^{3}\right)$. While NEMD is well suited for the study of short-timescale phenomena around the shock front, chemistry and phase transitions well behind the shock front remain almost completely unexplored due to these scaling difficulties.

This chapter presents a method that circumvents these difficulties by treating some aspects of the shock wave within continuum theory. [6] This method requires molecular dynamics 
simulation only of a small part of the shock wave at a given instant in time. The effects of the shock wave passing over this small molecular dynamics system are simulated by regulating the applied stress and energy that are obtained from a continuum theory description of the shock wave structure. Because the size of the molecular dynamics system is independent of the simulation time in this approach, the computational work required to simulate the shock is linear in the simulation time (computational work is of order $O\left(t_{\text {simulation }}\right)$ ), circumventing the scaling problems of NEMD. This multiscale approach attempts to constrain the molecular dynamics system to the same thermodynamic states that are found in the macroscopic shock wave, ensuring that thermodynamic path-dependent processes are captured correctly. As a benefit of following the correct thermodynamic pathway, it can be shown that this multiscale approach requires no a priori knowledge of the system phase diagram, metastable states, chemical reaction or phase transformation rates, or sound speeds. The method can also detect the presence of material instabilities that lead to the formation of double shock waves and simulate these double shock waves in an approximate fashion.

The first sections of this chapter are devoted to a description of the method and practical details for its implementation and utilization. Subsequent sections extend the method to the detection and simulation of double shock waves, which are ubiquitous in condensed matter. Example applications are presented for a Lennard-Jones atomic potential system (which can provide a description of solid Argon), an empirical potential model of crystalline silicon, and a tight-binding atomic potential for the chemically reactive explosive nitromethane $\left(\mathrm{CH}_{3} \mathrm{NO}_{2}\right)$.

\section{MULTI-SCALE MODEL DERIVATION}

We model the propagation of the shock wave using the 1D Euler equations for compressible flow, which neglect thermal transport.

$$
\begin{aligned}
& \frac{d \rho}{d t}+\rho \frac{\partial u}{\partial x}=0 \\
& \frac{d u}{d t}+\tilde{v} \frac{\partial p}{\partial x}=0 \\
& \frac{d \tilde{e}}{d t}+p \frac{d \tilde{v}}{d t}=0
\end{aligned}
$$

Here, $\rho$ is the density, $u$ is the local material velocity, $\tilde{v}=\frac{1}{\rho}$ is the specific volume, $p$ is the pressure, $\tilde{e}$ is the energy per unit mass, and complete time derivatives are $\frac{d f}{d t} \equiv \frac{\partial f}{\partial t}+u \frac{\partial f}{\partial x}$. We take the variables in these equations to be instantaneous (microscopic) variables that include thermodynamic fluctuations, i.e. thermodynamic fluctuations are not averaged over. These equations represent the conservation of mass, momentum, and energy respectively everywhere in the wave. No explicit terms account for thermal transport in these equations. The validity of this approximation is discussed in the section on thermal transport below. 
While the Euler equations are not rigorously applicable at elastic shock fronts which can be atomistically sharp in NEMD simulations, it will be shown that the correct dynamics will be approximated in these special regions. It is expected that these continuum equations provide a reasonable description of the shocked material in the region behind the shock front, after any very short length-scale phenomena occur at the shock front.

We seek solutions of the Euler equations which are steady in the frame of the shock wave moving at speed $\mathrm{v}_{\mathrm{s}}$ by making the substitution $(x, t) \rightarrow x-\mathrm{v}_{\mathrm{s}} t$. This substitution, and integration over $x$ yields a variation of the Hugoniot relations:

$$
\begin{aligned}
& u-u_{0}=\left(\mathrm{v}_{\mathrm{s}}-u_{0}\right)\left(1-\frac{\rho_{0}}{\rho}\right) \\
& p-p_{0}=\left(u_{0}-\mathrm{v}_{\mathrm{s}}\right)^{2} \rho_{0}\left(1-\frac{\rho_{0}}{\rho}\right) \\
& \tilde{e}-\tilde{e}_{0}=p_{0}\left(\frac{1}{\rho_{0}}-\frac{1}{\rho}\right)+\frac{\left(\mathrm{u}_{0}-\mathrm{v}_{\mathrm{s}}\right)^{2}}{2}\left(1-\frac{\rho_{0}}{\rho}\right)^{2} .
\end{aligned}
$$

Variables with subscripts 0 are the values before the shock wave, and we take $u_{0}=0$, i.e. the material is initially at rest in the laboratory frame. In the terminology of shock physics, Eq. (5) for the pressure is the Rayleigh line and Eq. (6) for the internal energy is the Hugoniot at constant shock velocity. The Hugoniot condition Eq. (6) is more commonly written as

$$
\tilde{e}-\tilde{e}_{0}=\frac{1}{2}\left(p+p_{0}\right)\left(\tilde{v}_{0}-\tilde{v}\right)
$$

by combining Eq. (5) with Eq. (6) to eliminate $\mathrm{v}_{\mathrm{s}}$. These equations apply to a system that has a time-independent steady-state in the reference frame moving at the shock speed $\mathrm{v}_{\mathrm{s}}$.

If the stress and energy of a molecular dynamics simulation can be constrained to obey Eq. (5) and Eq. (6), then the simulation proceeds through the same thermodynamic states that would occur in a steady shock. The goal of this section is to present a Lagrangian for the molecular dynamics system that performs these constraints. The fashion in which this constraint is done is not unique, and there may be other Lagrangians that perform the same basic constraint task with some different fluctuation or other properties. We choose the Lagrangian for the molecular dynamics simulation to be

$$
L=\frac{1}{2} \sum_{i} m_{i} \dot{\vec{r}}_{i}^{2}-\phi\left(\left\{\vec{r}_{i}\right\}\right)+\frac{1}{2} M Q \dot{\tilde{v}}^{2}+\frac{1}{2} M \frac{\mathrm{v}_{\mathrm{s}}^{2}}{\tilde{v}_{0}^{2}}\left(\tilde{v}_{0}-\tilde{v}\right)^{2}+M p_{0}\left(\tilde{v}_{0}-\tilde{v}\right)
$$

where $\phi$ is the potential energy, $M=\sum_{i} m_{i}$ is the total system mass, $Q$ is a mass-like parameter for the simulation cell size. The pre-shock material is taken to be at rest, i.e. $u_{0}=0$. All variables in Eq. (8) are instantaneous variables, i.e. they are not thermally averaged. Note that $Q$ has units of mass $^{2} /$ length $^{4}$. To enable simulations in systems with periodic boundary conditions, consider the use of the scaled coordinate transformations as in Ref. [11] and [7], 
$\vec{r} \equiv A \vec{s}$

$\dot{\vec{r}} \equiv A \dot{\vec{s}}$

where $A$ is a matrix containing the computational cell lattice vectors in columns and $\vec{s}$ is a column vector containing scaled coordinates relative to the computational cell. The values of the scaled coordinates range between 0 and 1 . Choosing velocities to be purely a function of $\dot{\vec{s}}$ without a term involving $\vec{s}$ provides a computational cell where the velocity of a particle is independent of its position. The real-space velocities within this coordinate system can be recovered through the relation, $\dot{\vec{r}}_{\text {real-space }}=A \dot{\vec{s}}+\dot{A} \vec{s}$.

For simplicity, assume that the computational cell is orthorhombic with box lengths $a_{x}, a_{y}$, and $a_{z}$. We take the shock to be propagating in the $x$ direction. Planar shocks are described by the 1D Euler equations so only $a_{x}$ is allowed to vary, providing a uniaxial strain condition. Computational cell dimensions transverse to the shock direction are fixed, as in NEMD simulations.

The Hamiltonian form of Eq. (8) is,

$$
H=\sum_{i, \alpha} \frac{P_{i, \alpha}^{2}}{2 m_{i} a_{\alpha}^{2}}+\phi\left(\left\{A \vec{s}_{i}\right\}\right)+\frac{P_{a_{x}}^{2}}{2 Q} \frac{M}{\left(a_{y} a_{z}\right)^{2}}-\frac{1}{2} M \mathrm{v}_{\mathrm{s}}^{2}\left(1-\frac{a_{x}}{a_{x, 0}}\right)^{2}-p_{0} a_{y} a_{z}\left(a_{x, 0}-a_{x}\right)
$$

where the momentum of particle $i$ in the direction $\alpha=\{x, y, z\}$ is $P_{i, \alpha}=m_{i} a_{\alpha}^{2} \dot{s}_{i, \alpha}$, momentum of the computational cell lattice vector $a_{x}$ is $P_{a_{x}}=\frac{Q}{M} \dot{a}_{x}\left(a_{y} a_{z}\right)^{2}$, and the value of $a_{x}$ in the preshock state is $a_{x, 0}$. In addition to the usual kinetic and potential terms associated with the atomic degrees of freedom, this Hamiltonian contains a degree of freedom associated with changes in the volume, $a_{x}$. The third term in Eq. (9) is a kinetic term for changes in volume and the last two terms represent an external potential for the volume.

The equation of motion for $P_{i, \alpha}$ is,

$\dot{P}_{i, \alpha}=-a_{\alpha} \frac{\partial \phi}{\partial r_{i, \alpha}}$

or,

$\ddot{s}_{i, \alpha}=-\frac{1}{m_{i} a_{\alpha}} \frac{\partial \phi}{\partial r_{i, \alpha}}-2 \frac{\dot{a}_{\alpha}}{a_{\alpha}} \dot{s}_{i, \alpha}$.

The equation of motion for $P_{a_{x}}$ is,

$$
\dot{P}_{a_{x}}=\sum_{i} \frac{P_{i, x}^{2}}{m_{i} a_{x}^{3}}-\frac{\partial \phi}{\partial a_{x}}\left(\left\{A \vec{s}_{i}\right\}\right)-\frac{M \mathrm{v}_{s}^{2}}{a_{x, 0}}\left(1-\frac{a_{x}}{a_{x, 0}}\right)-p_{0} a_{y} a_{z} .
$$

Multiplication of Eq. (12) by $\frac{1}{2}\left(a_{x, 0}-a_{x}\right)$ and substitution into the Hamiltonian yields, 


$$
\begin{aligned}
& H=\sum_{i, \alpha} \frac{P_{i, \alpha}^{2}}{2 m_{i} a_{\alpha}^{2}}+\phi\left(\left\{A \vec{s}_{i}\right\}\right)+\frac{P_{a_{x}}^{2}}{2 Q} \frac{M}{\left(a_{y} a_{z}\right)^{2}}-\frac{1}{2} M \mathrm{v}_{\mathrm{s}}^{2}\left(1-\frac{a_{x}}{a_{x, 0}}\right)^{2}-\frac{1}{2} p_{0} a_{y} a_{z}\left(a_{x, 0}-a_{x}\right) \\
& +\left[\frac{\partial \phi}{\partial a_{x}}\left(\left\{A \vec{s}_{i}\right\}\right)-\sum_{i} \frac{P_{i, x}^{2}}{m_{i} a_{x}^{3}}+\dot{P}_{a_{x}}\right] \frac{\left(a_{x, 0}-a_{x}\right)}{2}
\end{aligned}
$$

or,

$$
H=e+\frac{P_{a_{x}}^{2}}{2 Q} \frac{M}{\left(a_{y} a_{z}\right)^{2}}-\frac{1}{2} p_{0} a_{y} a_{z}\left(a_{x, 0}-a_{x}\right)-\frac{1}{2} p a_{y} a_{z}\left(a_{x, 0}-a_{x}\right)+\frac{1}{2} \dot{P}_{a_{x}}\left(a_{x, 0}-a_{x}\right)
$$

where the instantaneous energy $e$ is defined as,

$$
e \equiv \sum_{i, \alpha} \frac{P_{i, \alpha}^{2}}{2 m_{i} a_{\alpha}^{2}}+\phi\left(\left\{A \vec{s}_{i}\right\}\right)
$$

and the instantaneous uniaxial pressure $\left(-\sigma_{x x}\right.$ stress tensor component) is,

$$
p \equiv-\frac{1}{a_{y} a_{z}}\left[\frac{\partial \phi}{\partial a_{x}}\left(\left\{A \vec{s}_{i}\right\}\right)-\sum_{i} \frac{P_{i, x}^{2}}{m_{i} a_{x}^{3}}\right] .
$$

At $t=0$ when the system is in the pre-shock state, $a_{x}=a_{x, 0}$ and $P_{a_{x}}^{2}=0$ leading to, $H=e(t=0) \equiv e_{0}$. The Hamiltonian Eq. (13) can be rewritten,

$$
e-e_{0}=\frac{1}{2}\left(p+p_{0}\right)\left(v_{0}-v\right)-\dot{P}_{a_{x}} \frac{\left(a_{x, 0}-a_{x}\right)}{2}-\frac{P_{a_{x}}^{2}}{2 Q} \frac{M}{\left(a_{y} a_{z}\right)^{2}}
$$

where $v=a_{x} a_{y} a_{z}$ and $v_{0}=a_{x, 0} a_{y} a_{z}$ in this case. Time averaging of this equation yields the time average of the Hugoniot energy condition, Eq. (7), plus terms of order $1 / N$, where $N$ is the number of particles in the system, resulting from the average of the last two terms in Eq. (15), i.e.,

$$
\left\langle e-e_{0}\right\rangle=\left\langle\frac{1}{2}\left(p+p_{0}\right)\left(v_{0}-v\right)\right\rangle+O\left(\frac{1}{N}\right)
$$

The time averages of the last two terms in Eq. (15) can be shown equal using the virial theorem. [8] The degree of adherence of the simulation energy to the Hugoniot condition increases with an increasing number of particles in the system and increases with the duration of the simulation until equipartition of energy among all degrees of freedom is achieved.

Time averaging of Eq. (9) can also be shown to lead directly to the time-averaged version of Eq. (6) with one additional term of order $O(1 / N)$. This time-averaged microscopic Hugoniot relation differs from the Hugoniot relation calculated using time-averaged thermodynamic quantities by,

$$
\frac{\mathrm{v}_{\mathrm{s}}^{2}}{2} \frac{\left(\left\langle\tilde{v}^{2}\right\rangle-\langle\tilde{v}\rangle^{2}\right)}{\tilde{v}_{0}^{2}}
$$


which goes to zero in the limit of vanishing volume fluctuations.

Eq. (12) for the motion for the system volume can be expressed in terms of the instantaneous pressure, Eq. (14), as,

$\dot{P}_{a_{x}}=\ddot{a}_{x} \frac{Q}{M}\left(a_{y} a_{z}\right)^{2}=a_{y} a_{z}\left(p-p_{0}\right)-\frac{M \mathrm{v}_{\mathrm{s}}^{2}}{a_{x, 0}}\left(1-\frac{a_{x}}{a_{x, 0}}\right)$.

Upon time-averaging, $\left\langle\dot{P}_{a_{x}}\right\rangle \equiv \lim _{\tau \rightarrow \infty} \frac{1}{\tau} \int_{0}^{\tau} \dot{P}_{a_{x}}=\lim _{\tau \rightarrow \infty} \frac{1}{\tau}\left[P_{a_{x}}(\tau)-P_{a_{x}}(0)\right]=0$ since $P_{a_{x}}(t)$ is bounded, and this equation of motion reduces to the Rayleigh line Eq. (5),

$$
\left\langle p-p_{0}\right\rangle=\left\langle\mathrm{v}_{\mathrm{s}}^{2} \rho_{0}\left(1-\frac{\rho_{0}}{\rho}\right)\right\rangle \text {. }
$$

By choosing a small representative molecular dynamics sample of the shocked material, application of the Euler equations requires that macroscopic stress, thermal, and density gradients in the actual shock wave are negligible on the length scale of the molecular dynamics computational cell size. While the thermal energy is assumed to be evenly spatially distributed throughout the sample by the shock, thermal equilibrium within the internal degrees of freedom computational cell is not required.

Some physical intuition for the function of this constraint scheme can be achieved. The last two terms in the Hamiltonian Eq. (9) are potential energy terms for the motion of the computational cell volume, or $a_{x}$ in this case. The energy associated with the shock is initially contained entirely in the potential energy of $a_{x}$ at $t=0$ because $\dot{a}_{x}(t=0)=0$. When the simulation begins, $a_{x}$ oscillates in a potential that is determined by the last two terms and the second term in Eq. (9). These oscillations are damped through coupling with the atomic degrees of freedom resulting from the second term in Eq. (9), i.e. energy flows from the volume degrees of freedom into the atomic degrees of freedom. Since there are many more atomic degrees of freedom (typically at least 100 in simulations we have performed) than volume degrees of freedom (there are two: $a_{x}$ and $P_{a_{x}}$ ), this flow of energy is irreversible.

This heat flow continues until the volume oscillations are damped and statistical equipartition of energy among all the degrees of freedom is achieved.

The time-dependent properties of the molecular dynamics simulation are characteristic of a material element flowing through the shock wave, within the approximations made in the derivation of the method. Therefore, the spatial profile of the simulated shock wave can be reconstructed by calculating the position of a material element $x$ at time $t$,

$$
x(t)=-\int_{0}^{t}\left(\mathrm{v}_{\mathrm{s}}-u\left(t^{\prime}\right)\right) d t^{\prime}
$$

where $u(\rho(t))$ is given by Eq. (4). In this fashion, the spatial dependence of all quantities in Eqs. (4), (5), and (6) can be determined for the steady shock wave. 


\section{STABILITY OF SIMULATED WAVES}

The molecular dynamics constraint technique presented in the previous section is designed to simulate steady solutions of the Euler equations but there is no guarantee that all of the simulated solutions are physical. Some steady solutions are characterized by unbounded volume expansion, and others may not be the particular shock wave solutions desired. This section defines mechanical stability conditions that characterize shock waves and then shows that the molecular dynamics constraint technique naturally takes the system through states that satisfy these stability conditions.

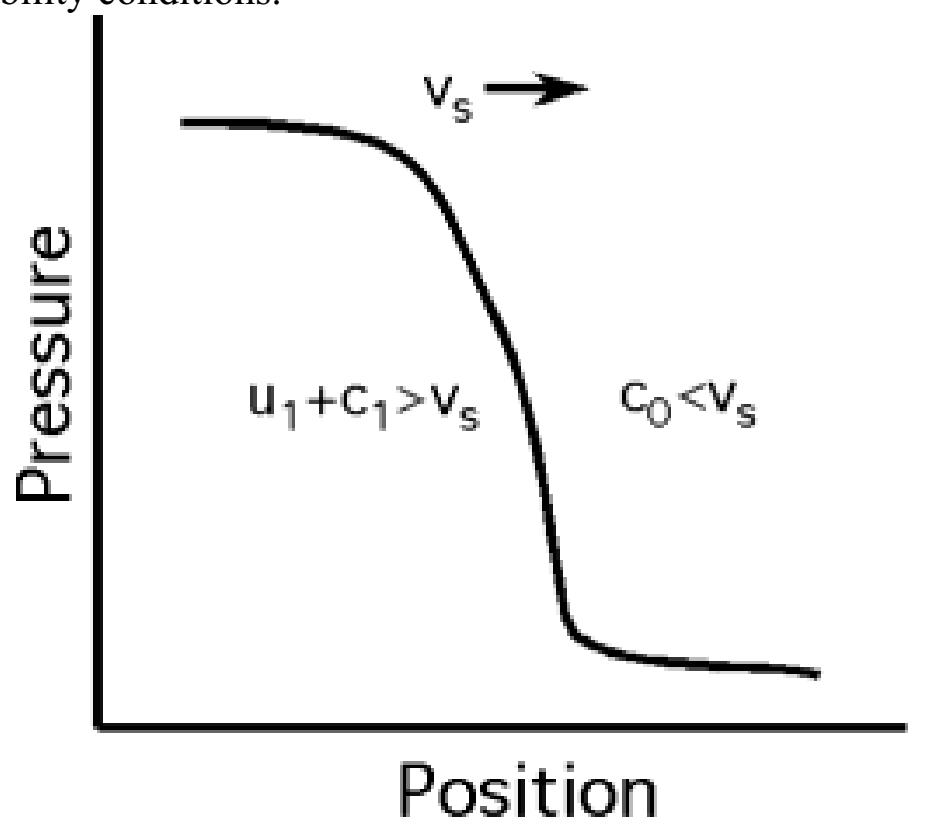

Figure 1. Schematic of a shock wave showing the conditions on sound speed, particle velocity, and shock speed required for mechanical stability in front of and behind the shock front.

There are two criteria for the mechanical stability of a shock wave. [9] The first criterion requires $\mathrm{V}_{\mathrm{s}}>c_{0}$, where $c_{0}$ is the speed of sound in the pre-shock material. The second criterion requires $u_{1}+c_{1}>\mathrm{v}_{\mathrm{s}}$, where the subscript 1 denotes the post-shock state.

The condition that $\mathrm{v}_{\mathrm{s}}>c_{0}$ can be motivated physically by considering the propagation of sound waves in front of the shock. If $\mathrm{v}_{\mathrm{s}}<c_{0}$, then pressure waves from behind the shock front can propagate out in front, resulting in an increase of the shock front width and eventual decay of the shock front. The stability condition behind the shock that $u_{1}+c_{1}>\mathrm{v}_{\mathrm{s}}$ can be motivated by considering the case where $u_{1}+c_{1}<\mathrm{v}_{\mathrm{s}}$. In this case, the shock front propagates faster than the speed of sound waves behind it. Compressive energy (in the form of a piston, etc.) behind the shock cannot reach the shock front, resulting in a decay of the shock pressure and eventual dissipation.

The equation of motion for the volume Eq. (12) can be shown to constrain the molecular dynamics system to thermodynamic states that satisfy the conditions for mechanical shock stability. As an example system, consider a shock from state A to state E of Figure 2. Figure 2 shows Rayleigh lines on a hypothetical shock Hugoniot. Eq. (12) indicates that volume increases or decreases depending on the relation between the stress of the molecular dynamics system (approximately given by the Hugoniot line in Figure 2) and that of the Rayleigh line 
stress (given by the straight lines in Figure 2). When the simulation begins at state A, shock compression will occur if the Rayleigh line is above the Hugoniot in pressure-volume space and the volume is initially slightly on the compressed side of the volume of state A. The slope of the Rayleigh line is $-v_{S}^{2} / \tilde{v}_{0}^{2}$. The Hugoniot and isentrope have a first-order tangent at point A, [9] providing a Hugoniot slope of $-c_{0}^{2} / \tilde{v}_{0}^{2}$ at state A. Therefore the stability condition $\mathrm{v}_{\mathrm{s}}>c_{0}$ must be satisfied at point $\mathrm{A}$ if compression proceeds up along the Rayleigh line since the slopes obey the condition $-\mathrm{v}_{\mathrm{S}}^{2} / \tilde{v}_{0}^{2}<-c_{0}^{2} / \tilde{v}_{0}^{2}$ which implies $\mathrm{v}_{\mathrm{s}}>c_{0}$. If the shock speed is chosen such that $\mathrm{v}_{\mathrm{s}}<c_{0}$, then point $\mathrm{A}$ is a stable point of Eq. (12) and no compression will occur.

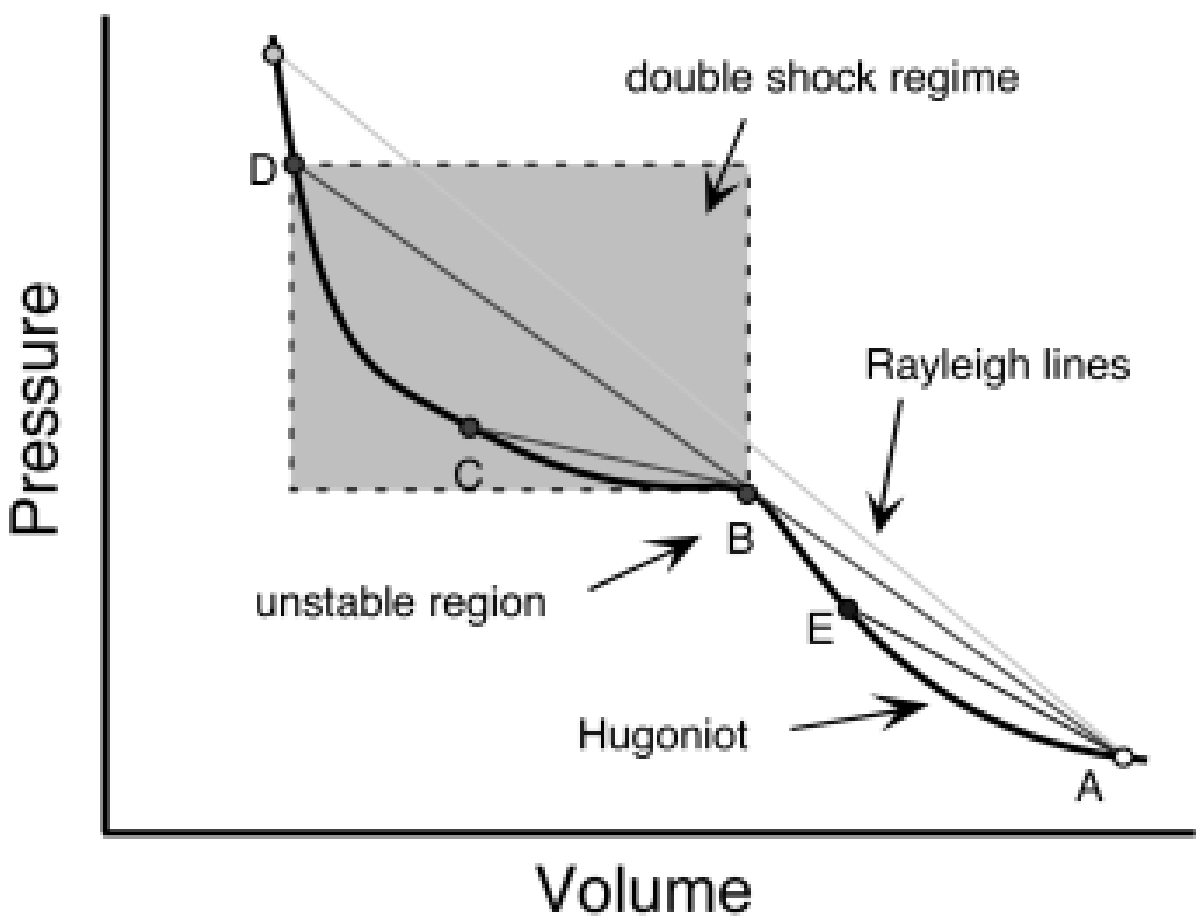

Figure 2. Rayleigh lines on a hypothetical Hugoniot. States on the Hugoniot outside of the gray box can be reached with a single Rayleigh line, or a single shock wave. States on the Hugoniot inside the gray box require two Rayleigh lines to be reached, or two shock waves.

The volume equation of motion Eq. (12) has stable points (where compression stops) at states where the Rayleigh line intersects the Hugoniot and the Rayleigh line slope magnitude is less than the Hugoniot slope magnitude. Point B in Figure 2 is an example of such a state. It can be shown that this condition on the Rayleigh line and Hugoniot slopes requires $u_{1}+c_{1}>\mathrm{v}_{\mathrm{s}}$ which is the stability condition behind the shock front. Ref. [9] contains an outline of this proof. Therefore the constraint Eq. (12) has stable points only where the shock wave mechanical stability conditions are met.

Prior knowledge of the local sound speeds is not required when beginning a simulation. If compression occurs at state $\mathrm{A}$, then $\mathrm{v}_{\mathrm{s}}>c_{0}$. If compression stops at state $\mathrm{B}$, then $u_{1}+c_{1}>\mathrm{v}_{\mathrm{s}}$. Note that as a consequence of the instability at point A of Eq. (12), runaway expansion on the tensile strain side of state $\mathrm{A}$ is also a valid solution of the steady state Euler equations. Such an expansion solution may have physical significance if there exists a larger volume where 
Eq. (12) has a stable solution. Such solutions are expansion shocks and can be observed in materials where the Hugoniot has the property that $\left.\frac{\partial^{2} p}{\partial v^{2}}\right|_{\text {Hugoniot }}<0$ in some region. However, this chapter focuses on the compressive shock solutions. To allow only compressive shock solutions, a variety of techniques can be used to bias the simulation at point $\mathrm{A}$ to proceed along the compressive branch of the Rayleigh line rather than the expansive branch. These techniques are discussed within the section on Computational Details.

\section{NEGLECT OF THERMAL TRANSPORT}

Under some circumstances, the flow of heat within a shock can have an effect of the thermodynamic states the shocked material exhibits. Heat flow can be mediated by a variety of mechanisms including phonons, electrons, and photons. [10] Radiative heat flow is important only the most extreme shocks where temperatures are generally above a few thousand Kelvin. Electron mediated heat flow is also important above a few thousand Kelvin in insulating materials, but can be important at much lower temperatures in materials with a small electronic bandgap or in metals. For shocks in insulating materials where the temperatures are less than a few thousand Kelvin, phonons are the primary medium for heat flow.

The Euler equations do not contain any explicit terms related to the flow of heat. Therefore, care should be exercised when applying the method presented in this chapter to situations where significant thermal gradients exist. Photon and electron mediated heat flow propagates orders of magnitude faster than typical shock speeds. These heat flow mechanisms can cause pre-heating of the material in front of the shock among other effects. While all of the thermodynamic simulated states within the shock are not necessarily captured correctly in the molecular dynamics simulation correct in this case, the final thermodynamic state of the shock wave still obeys the Equations (4), (5), and (6) even with heat flow because these equations are based purely on the conservation of mass, momentum, and energy across the entire shock wave structure. Heat flow plays a role within the shock front structure, but does not affect these conservative properties across the shock wave. Therefore, the method presented here can still predict the correct final state of the shock if the assumption is made that the final state is not sensitive to the particular thermodynamic path through which it is reached.

While the treatment of phonon mediated heat flow is not strictly accounted for in this method, a rigorous statement can be made in this case. The mechanical shock stability conditions of the previous section can be used to show that phonon-mediated heat flow cannot occur in the forward direction through the shock front. Phonon propagation speeds in a particular direction are equal to or less than the sound speed in that direction. Therefore the stability condition $\mathrm{v}_{\mathrm{s}}>c_{0}$ in front of the shock front prevents heat behind the front from propagating out in front to pre-heat. A related statement can be made regarding the propagation of heat from a plastic wave to an elastic wave in a double shock scenario. (Double shock waves are discussed in subsequent sections.) In this scenario, continuum theory requires that there is a region in the shock where $\mathrm{v}_{\mathrm{s}}=c_{1}+u_{1}$. This region marks the 
boundary between the first and second shock waves, and represents a region where heat is not allowed to flow forward from the second shock wave into the first shock wave. Therefore, the temperature of an elastic shock wave is unaffected by the temperature of the subsequent plastic shock wave within continuum theory.

The importance of the neglect of phonon-mediated heat flow can be estimated using a diffusion model,

$\frac{\partial T}{\partial t}=D \frac{\partial^{2} T}{\partial x^{2}}$

where $T$ is the temperature and $D$ is the thermal diffusivity. Consider the case where the temperature has a steady profile in a reference frame moving at the shock speed, i.e. $T(x, t)=T\left(x-\mathrm{v}_{\mathrm{s}} t\right) .[10]$ Then Eq. (18) becomes,

$-\mathrm{v}_{\mathrm{s}} \frac{\partial T}{\partial x}=D \frac{\partial^{2} T}{\partial x^{2}}$

which has solutions of the form

$$
T=\left(T_{1}-T_{0}\right) \exp \left(-\frac{\mathrm{v}_{\mathrm{s}}}{D}\left(x-x_{0}\right)\right)+T_{0}
$$

where $T_{0}$ and $T_{1}$ are the temperatures in front of and behind the shock, respectively, and $x_{0}$ is the location of the point where the final post-shock temperature $T_{1}$ is reached. This system is equivalent to a constant temperature source with temperature $T_{1}$ (i.e., the post-shock material), moving at the shock speed. The characteristic length that the post-shock thermal energy diffuses forward in the propagation direction is given by,

$\Delta x \sim \frac{D}{\mathrm{v}_{\mathrm{s}}}$.

This characteristic length can be compared to the characteristic length of temperature increase for a simulation using the method presented in this chapter. The latter can be determined using the time dependence of the temperature and Eq. (17). Heat conduction is not expected to be important if the length given by Eq. (19) is substantially less than the characteristic length scale for temperature increase in the simulation.

Typical values of $\Delta x$ range from around tens of $\mathrm{nm}$ for metals like gold to a few Angstroms or less for insulating materials. Molecular solid energetic materials like nitromethane (to be discussed later) fall into the latter category where thermal transport is expected to play little role.

The addition of heat flow mechanisms to the continuum equations utilized here results in a breakdown of the locality of Equations (4), (5), and (6), i.e., the thermodynamic variables at a given point are not purely a function of other variables at that point. For example, the temperature of material in front of the shock will depend on the temperature behind the shock when radiative or electronic heat conduction mechanisms are at play. We speculate that it may be possible to extend the method presented in this chapter to solve for steady shock waves with heat flow by utilizing an iterative procedure. For example, the temperature profile 
determined during an initial simulation can be time-evolved using a relevant heat diffusion equation to obtain a temperature profile to be enforced for a subsequent simulation, and so on. If such an iterative procedure is carried out, a steady propagating wave with heat flow may be determined if the iterations converge on a time-dependence for the various thermodynamic quantities.

\section{COMPUTATIONAL DETAILS}

This section presents and discusses the practical issues associated with use of the constraint technique presented in this chapter. Some of these key issues include the need for energy conservation, techniques for ensuring the simulation initially proceeds along the compressive branch of the Rayleigh line, criteria for the choice of the empirical mass-like parameter $Q$ in Eq. (8), and criteria for the choices of computational cell size and simulation duration.

\subsection{Adherence to constraints}

Unlike many popular molecular dynamics thermostating techniques, the technique presented in this chapter is conservative with respect to energy. The atomic degrees of freedom are coupled to the volume degree of freedom, which has a fixed amount of energy at the start of the simulation. In this respect, this technique is related to the Andersen technique for constant pressure simulations. [11] Therefore, it is important to ensure that the integration time-step is chosen to be sufficiently small to conserve the Hamiltonian value, Eq. (9). We utilize a Verlet-based integration algorithm to integrate the equations of motion for the volume (Eq. (16)) and the atoms (Eq. (11)). In the case of atomic equations of motion, the use of scaled coordinates results in a velocity dependent force which must be computed using velocities determined from the atomic trajectories within this Verlet scheme, leading to a suboptimal integration algorithm. While this algorithm can be made to conserve energy sufficiently by choosing a sufficiently small time step, a different integration algorithm would enable use of a smaller time-step. An example of a higher-order accuracy integration algorithm can be found in Ref. [12]

Figure 3 shows the time-dependence of various temperatures for an example simulation of an elastic-plastic shock in the [110] direction in an approximately cubic perfect 25688 atom face centered cubic Lennard-Jones crystal. This computational cell size is large enough to prevent artificial influence of the periodic boundary conditions on the deformation mechanisms inside the computational cell. [13] In terms of the standard Lennard-Jones potential parameters, the initial volume per atom $\frac{v_{0}}{\sqrt{2} \sigma^{3}}=0.68$, initial stress $p_{0}=0$, and initial temperature $k_{B} T_{0} / \varepsilon=0.01$ with shock speed $\frac{\mathrm{v}_{\mathrm{s}}}{c_{0}}=1.87$ where the longitudinal sound speed in the [110] direction $c_{0}=9.5$. [13] To aid in physical intuition for some of the Lennard-Jones simulations presented in this chapter, we have utilized parameters for Argon: $k_{B} \varepsilon=119.8 \mathrm{~K}, \sigma=0.3405 \mathrm{~nm}$ and mass $m=40$ atomic mass units. The Lennard-Jones simulations were performed using the spline potential of Ref. [14] to prevent numerical errors associated with a discontinuous potential at the cutoff. The spline parameters for this 
potential were chosen as in Ref. [14]. The Lennard-Jones simulations presented in this chapter utilized a timestep of $1.15 \times 10^{-4}$ LJ time units, which resulted in conservation of energy to $10^{-3} \varepsilon$ /atom for all of these simulations.

At the top of Figure 3 is the temperature of the atoms, showing the transition from elastic compression to plastic compression around $10 \mathrm{ps}$. The middle plot gives the temperature of the strain degree of freedom divided by the total number of degrees of freedom in the simulation. The peak strain degree of freedom temperature at the start of the simulation (about $17 \mathrm{~K}$ ) is approximately the amount of irreversible temperature increase the shock provides to the atomic degrees of freedom. The middle plot of Figure 3 shows the temperature of the strain degrees of freedom decrease with time as equipartition is approached. The bottom plot shows the temperature deviation from the initial Hamiltonian energy, Eq. (9), showing good energy conservation.

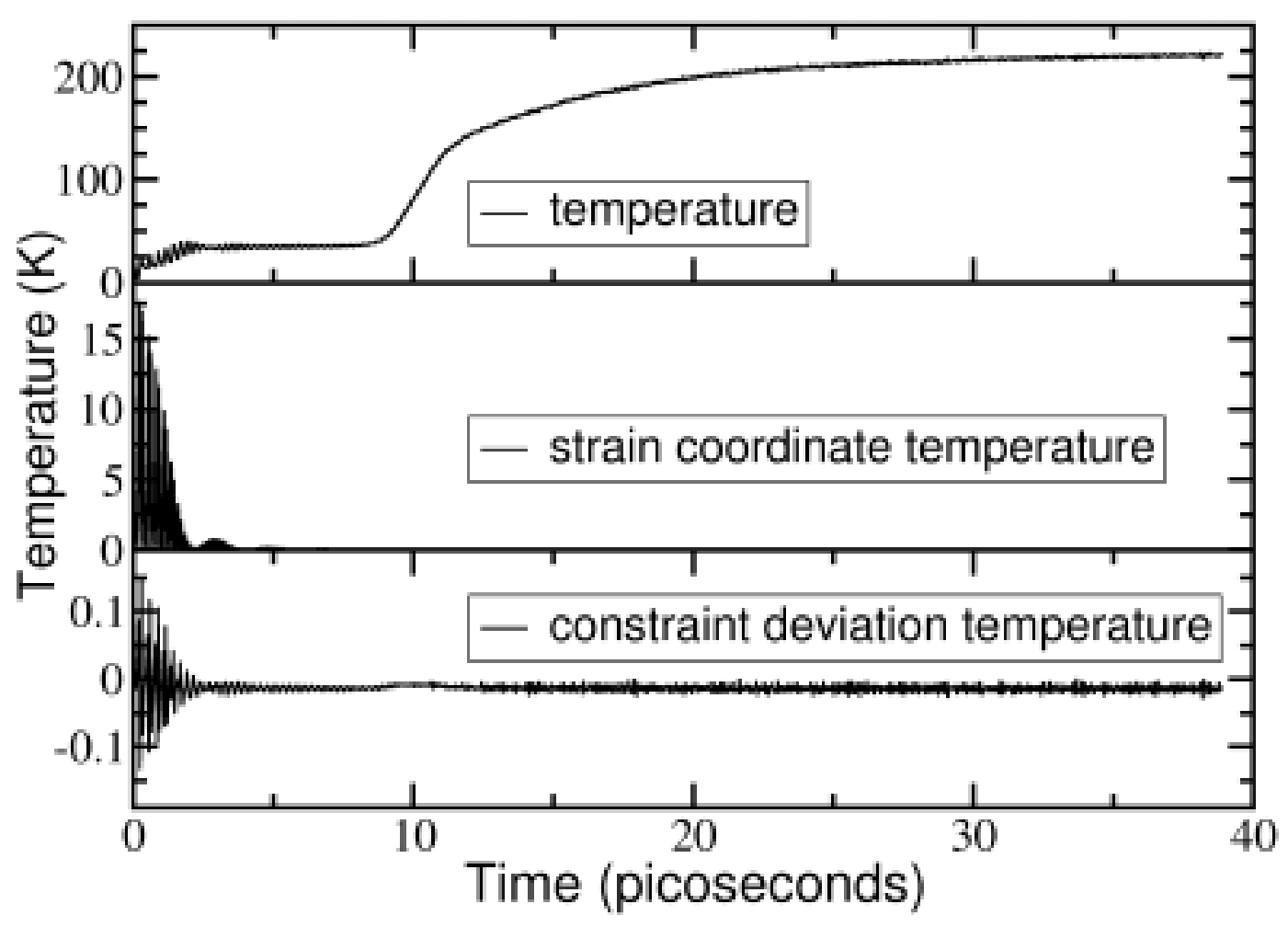

Figure 3: The time-dependence of various temperatures for an example simulation of an elastic-plastic shock in the [110] direction in a perfect 25688 atom face-centered cubic Lennard-Jones crystal. At top is the temperature of the atoms, showing the transition from elastic compression to plastic compression around $10 \mathrm{ps}$. The middle plot gives the temperature of the strain degrees of freedom divided by the total number of degrees of freedom in the simulation, showing the amount of irreversible energy the shock transfers to the atomic degrees of freedom. The bottom plot shows the temperature deviation from the initial Hamiltonian energy, Eq. (9), showing good energy conservation. 
Figure 4 shows the time-dependence of the temperature, uniaxial stress in the shock propagation direction, and volume for a $2.8 \mathrm{~km} / \mathrm{sec}$ shock in the [111] direction in a perfect 23400 atom Lennard-Jones crystal. The initial volume is $0.03851 \mathrm{~nm}^{3} /$ atom and initial temperature is $10 \mathrm{~K}$ with zero initial pressure. The initial pressure for this and all other simulations in this chapter was obtained by averaging over the instantaneous pressure of a constant volume simulation for some duration. Figure 4 shows initial compression to the elastically strained state for the first 2 picoseconds. While the system is elastically compressed, slow changes can be seen in the volume and stress. After 2 picoseconds, plastic deformation and further compression occurs. This deformation is characterized by initial fast changes in temperature, stress, and volume followed by a slower relaxation period. While the characterization of compression as either elastic or plastic plays no role in the molecular dynamics Hamiltonian, it is possible to determine the nature of the compression by monitoring the radial distribution function, visual inspection of the computational cell, or other means. The ab-initio character of the multi-scale method requires no knowledge of the nature of any plastic deformation mechanism or chemical reactions that occur in the system.

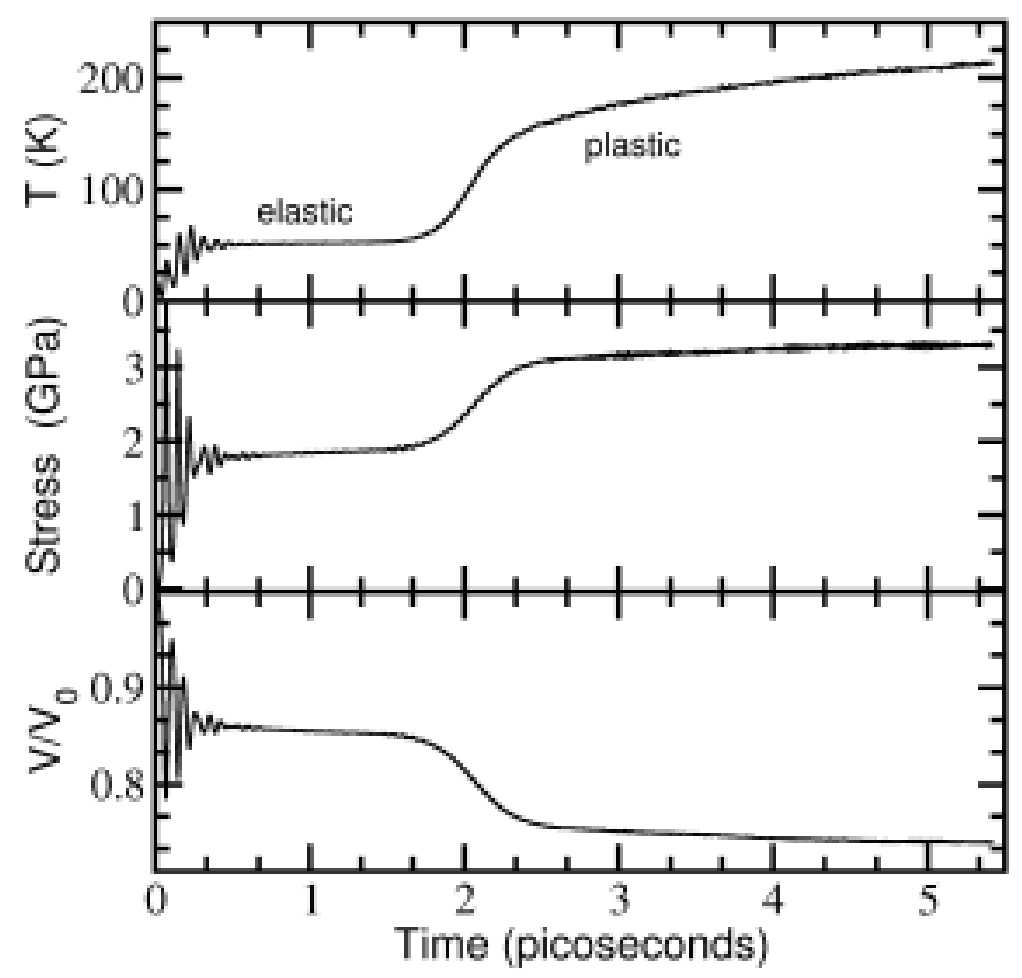

Figure 4: Time-dependence of temperature, uniaxial stress in the shock propagation direction, and volume calculated for an elastic-plastic shock in the [111] direction of a perfect Lennard-Jones crystal. After initial elastic compression, plastic deformation occurs around 2 picoseconds into the simulation. Lennard-Jones potential parameters have been chosen for Argon. See text for details. 
Figure 4 shows that initial elastic compression is characterized by oscillations of the volume. These volume oscillations are damped within about 5 oscillations in this case. The damping of volume oscillations occurs by transfer of energy from the strain degrees of freedom to the atomic degrees of freedom, and the atomic temperature can be seen to increase while this process occurs.

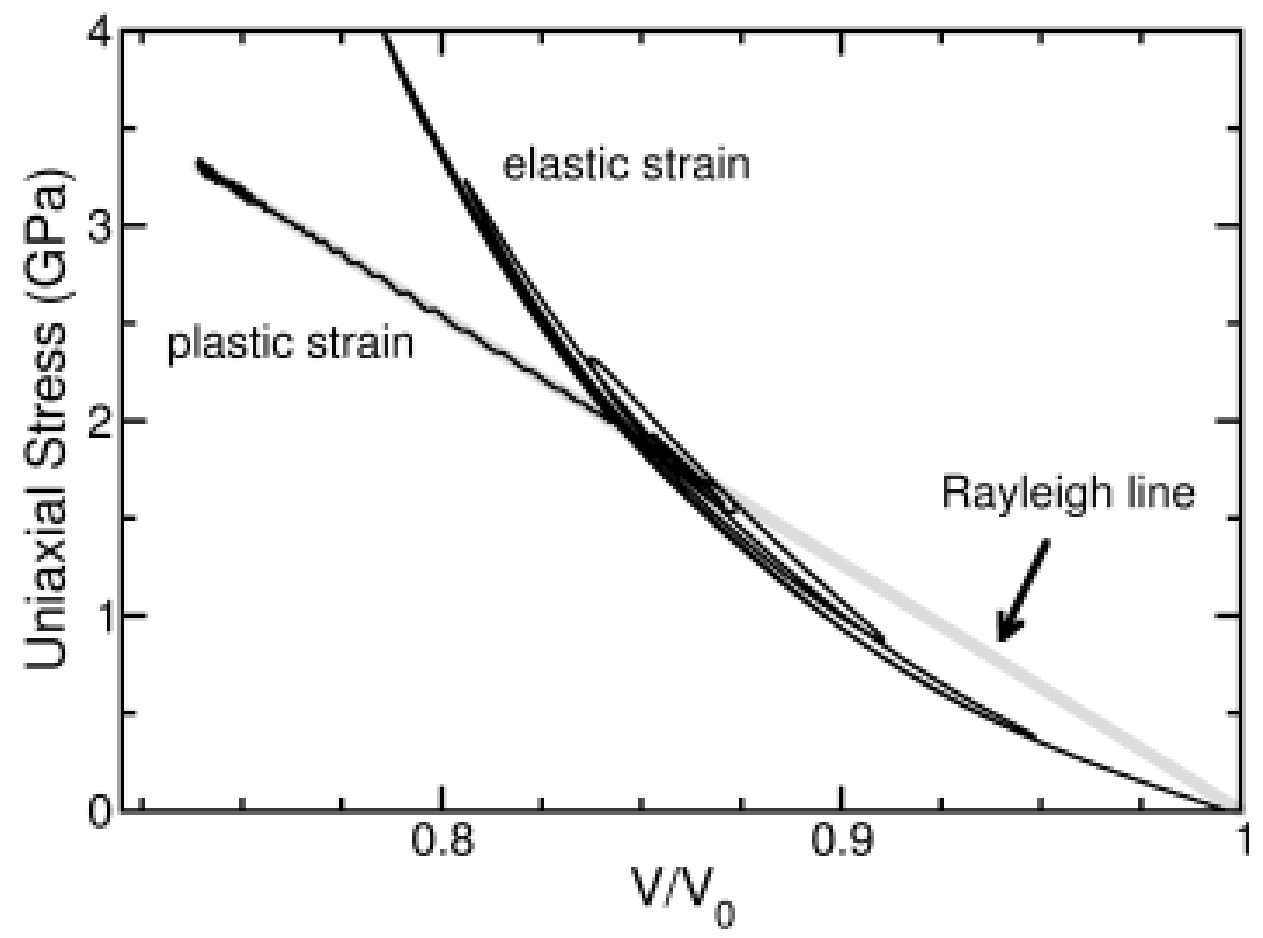

Figure 5: Uniaxial stress versus volume for an overdriven [111] direction shock simulation in a perfect Lennard Jones crystal. The gray line is the Rayleigh line, or constraint line provided by the volume equation of motion Eq. (16). The black line is the actual path of the simulation. The volume begins the simulation at $V / V_{0}=1$ and subsequently undergoes elastic oscillations around $V / V_{0} \approx 0.85$. As the amplitude of these oscillations decays with time, the simulation trajectory approaches the Rayleigh line. After the oscillations have decayed away, plastic deformation and further compression occur. During this slower plastic wave, the simulation trajectory closely follows the Rayleigh line, ensuring the correct sequence thermodynamic states are sampled.

Figure 5 shows the uniaxial stress versus volume for an overdriven [111] direction shock simulation in a perfect Lennard Jones crystal. The gray line is the Rayleigh line, or constraint line provided by the volume equation of motion Eq. (16). The black line is the actual path of the simulation. The volume begins the simulation at $V / V_{0}=1$ and subsequently undergoes elastic oscillations around $V / V_{0} \approx 0.85$. As the amplitude of these oscillations decays with time, the simulation trajectory approaches the Rayleigh line. After the oscillations have decayed away, plastic deformation and further compression occur. During this slower plastic 
wave, the simulation trajectory closely follows the Rayleigh line, ensuring the correct sequence thermodynamic states are sampled.

\subsection{Choice of parameter $Q$}

The observed initial elastic oscillations are of questionable physical significance. The damping rate of these oscillations is determined by the degree of coupling between the strain degrees of freedom and the atomic degrees of freedom. This coupling is determined by a variety of factors including the nature of the atomic potential in Eq. (9) and the magnitude of the mass-like parameter $Q$. The degree of coupling constitutes an effective viscosity. Oscillations are longer-lived for perfect crystalline systems at very low temperatures ( $1 \mathrm{~K}$ for Argon), where 100 or more oscillations can occur. In this case, internal degrees of freedom are unavailable for transfer of energy from the strain degrees of freedom due to high symmetry conditions. Initial volume oscillations can also be only 1 or 2 oscillations in other systems like molecular solids at room temperature.

The magnitude of $Q$ and the equation of state of the molecular dynamics system determine the frequency of the initial elastic oscillations in Figure 4. Figure 6 shows the timedependence of the volume for three simulations of $2.2 \mathrm{~km} / \mathrm{sec}$ shock waves in the [110] direction of a perfect 1400 atom nearly cubic Lennard-Jones crystal at about $1 \mathrm{~K}$. Each simulation was performed with a different mass-like parameter $Q$. If $Q$ is chosen too large (top panel), long-lived oscillations can result. If $Q$ is chosen too small (bottom panel) large amplitude oscillations that do not decay with time can result. An optimal value of $Q$ results in fast decay of volume oscillations. We find that values of $Q$ that provide fast volume oscillation damping result in volume oscillation frequencies that are resonant with internal vibrational degrees of freedom, i.e. the volume oscillation frequencies fall within the vibrational density of states of the atomic system. The number of oscillations required for equilibration in Figure 6 is significantly enhanced by the extremely low initial $1 \mathrm{~K}$ temperature and perfect crystallinity.

Since the elastic oscillations are typically short-lived (representing a small fraction of the duration of the entire simulation because most of the time is spent in the plastic wave) they can generally be overlooked as long as no unphysical irreversible chemistry or plastic deformation occurs during deviations from the Rayleigh stress conditions in these oscillations. For example, plastic deformation may occur during overcompression periods if the timescale for volume oscillations is sufficiently slow. The choice of parameter $Q$ should therefore be chosen to provide oscillations of sufficiently fast timescale to prevent any unphysical chemistry or plastic deformation from occurring during the oscillation damping process.

Figure 5 shows that the Rayleigh line is closely followed after the initial volume oscillations damp. Note that the timescale for plastic deformation in Figure 3 and Figure 4 is independent of the empirical parameter $Q$ since the strain in this regime changes on a timescale much slower than the resonant volume oscillation frequency determined by $Q$.

The scaled coordinate scheme applies strain uniformly throughout the computational cell, which is typically several lattice units or more in each dimension. The volume degrees of freedom may therefore be poorly coupled into very short spatial wavelength phonons which may be important in transfer of energy. Shock fronts in NEMD simulations in perfect crystals 
can possess thickness as short as a few atomic spacings. However, shock wave experiments typically involve polycrystalline materials and non-planar shocks so that shock front thickness is likely to be far greater than the atomic length scale. The latter observation lends some validity to the approximation of uniform strain across the computational cell.

As an alternative to simulating elastic waves, the initial state of the simulation method described here can in principle be obtained directly from an NEMD simulation. NEMD simulations are well suited to simulating shock fronts with high spatial strain gradients for relatively short periods of time. The method presented in this chapter is well suited to reproducing long timescale dynamics behind the shock front where strain gradients are not appreciable. Therefore these two complementary methods could be combined by taking the input computational cell from some point in an NEMD simulation behind the shock front where strain and other gradients have sufficiently relaxed. A less ambitious method to damp initial elastic oscillations may be to utilize a modified Hamiltonian containing additional terms to provide enhanced coupling between volume and atomic degrees of freedom. The additional viscosity can be tuned to prevent elastic oscillations. We have not utilized these approaches to simulation initialization in this chapter.

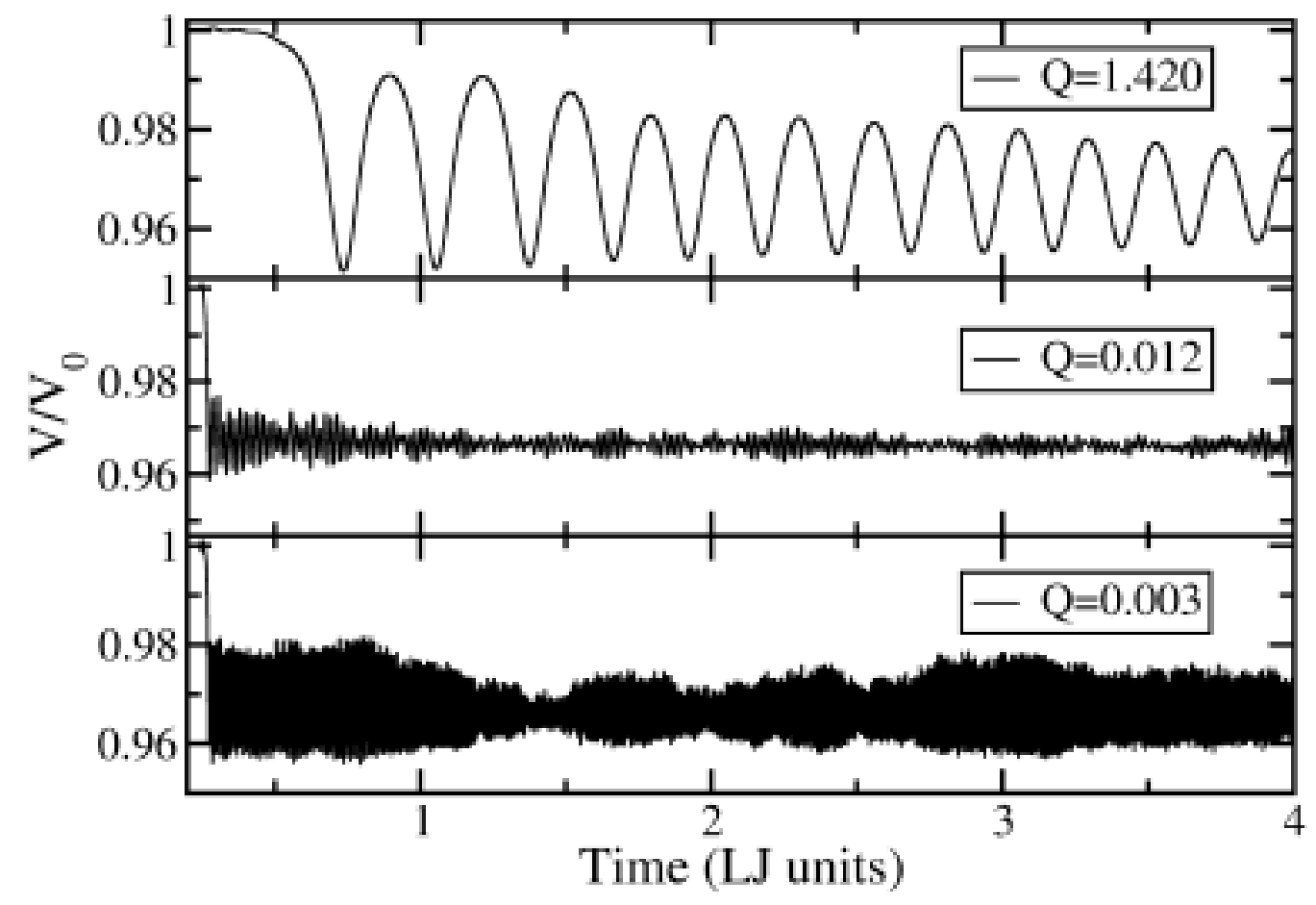

Figure 6. Depicted is the time-dependence of the volume for three simulations of $2.2 \mathrm{~km} / \mathrm{sec}$ shock waves in the [110] direction of a perfect 1400 atom Lennard-Jones crystal. Each simulation was performed with a different mass-like parameter $Q$ in Eq. (9), given here in reduced Lennard-Jones units. If $\mathrm{Q}$ is chosen too large (top panel), long-lived oscillations can result. If $Q$ is chosen too small (bottom panel) large amplitude oscillations that do not decay with time can result. An optimal value of $Q$ results in fast decay of volume oscillations. 


\subsection{Initialization bias for compressive shocks}

Another practical issue associated with the use of this simulation technique is biasing the instability of the starting point. As discussed in the section on stability, as long as the shock speed exceeds the local sound speed, the volume equation of motion Eq. (16) can either force compression or expansion of the volume. While both of these steady solutions can potentially have physical significance, the solutions we focus on in this chapter are the compressive, shock-like solutions. Therefore some technique is required for biasing the initial instability so that only compression occurs. Note that this is simply a selection of the particular type of steady solution to be simulated (compressive shock versus expansion shock) and does not represent nor require an empirical parameter or extra degree of freedom.

There are a wide variety of techniques that can be utilized to bias the volume equation of motion to yield only compressive shock solutions. Here we present two techniques that we have implemented for various molecular dynamics systems. One of these techniques involves modifying the Hamiltonian to apply a constant external pressure at $p_{0}$ when $v>v_{0}$. In this case, the volume equation of motion (Eq. (16)) becomes,

$$
\dot{P}_{a_{x}}=\ddot{a}_{x} \frac{Q}{M}\left(a_{y} a_{z}\right)^{2}=a_{y} a_{z}\left(p-p_{0}\right)-\theta\left(a_{x, 0}-a_{x}\right) \frac{M \mathrm{v}_{\mathrm{s}}^{2}}{a_{x, 0}}\left(1-\frac{a_{x}}{a_{x, 0}}\right)
$$

where $\theta$ is the Heaviside function. In this fashion, if a thermal fluctuation at the start of the simulation increases the volume, the system is forced back to a condition where $v<v_{0}$ where irreversible compression will occur. Another technique for ensuring compression occurs is to compressively strain the system a small amount at the start of the simulation. Some initial compressive strain provides an initial compressive force in Eq. (16) preventing expansion. Both of these techniques cause some small deviation from the conserved quantity given by Eq. (9), but we find that the magnitude of this deviation is negligible when compared with other errors like numerical integration errors. We find that the stress biasing technique of Eq. (20) works best for systems with small numbers of atoms. Thermal fluctuations can be large in such systems, and an appreciable strain can be required to utilize the strain bias technique. We have utilized the strain bias technique for all the Lennard-Jones simulations presented in this section, with an initial compressive strain of typically $10^{-3}$ to $10^{-4}$.

\subsection{Computational cell size}

Of importance in using this molecular dynamics technique and other molecular dynamics techniques that utilize periodic boundary conditions are issues with artificially-induced correlations due to the finite size of the computational cell. Artificial phase transitions or other dynamics can be observed when the computational cell dimensions are sufficiently small to allow a particle to interact with its (correlated) periodic image. Artificial effects can be circumvented by making the computational cell sufficiently large that periodic atomic images are separated by a distance greater than the atomic correlation length in the material.

In addition to periodic image interaction effects, there is an additional factor in choosing the computational cell size that must be considered when using this shock molecular dynamics technique. The connection to continuum theory is based on the assumption that the simulated material element (molecular dynamics system) is sufficiently small that stress, 


\section{4}

density, and energy density in the shock wave do not vary appreciably across the length scale of computational cell. An alternative statement of this condition is that,

$\dot{a}_{x}<<c$

where $c$ is the sound speed of the material within the computational cell and $\dot{a}_{x}$ is the rate of change of the computational cell dimension in the shock propagation direction, using notation from the previous sections. If Eq. (21) holds, sound waves are able to equilibrate gradients in stress, density and energy density within a material element of the computational cell dimensions while the dimensions change. This condition is not unlike that required for adiabatic or reversible evolution of a material element. Eq. (21) provides a limit on the maximum computational cell size as a function of the strain rate.

Planar elastic waves in NEMD simulations in perfect crystals at low temperatures can exhibit considerable strain rates with spatial strain gradients that are exist across the atomic length scale. Within such waves, the condition provided by Eq. (21) breaks down for all but computational cell sizes with atomic scale dimensions. Breakdown of the condition Eq. (21) can result in thermodynamic conditions within the computational cell that may not exist in the shock. However, we utilize computational cells much larger than the atomic scale even for elastic waves because we find that the end state of elastic waves is insensitive to the particular thermodynamic pathway through which it was reached. Satisfaction of Eq. (21) is of greatest concern during plastic deformation or chemical reactions, where the thermodynamic pathway of the computational cell can have an effect of the states of matter formed. Eq. (21) is generally easier to satisfy for a large computational cell in these waves because the strain rates tend to be considerably smaller than those at elastic wave fronts.

Since gradients in stress, density, and energy density tend to decrease in magnitude with distance behind the shock wave, Eq. (21) is expected to become valid at some point behind the shock front and hold thereafter. Materials with relatively short atomic correlation lengths, like molecular solids, can be simulated with smaller computational cells making satisfaction of Eq. (21) possible with larger strain gradients. The peak strain rate during plastic deformation in Figure 4 (around $2 \mathrm{ps}$ ) has $\dot{a}_{x} \approx 1 \mathrm{~km} / \mathrm{sec}$ which is in marginal satisfaction of Eq. (21) because the stability condition $c+u>\mathrm{v}_{\mathrm{s}}$ implies $c>2.3 \mathrm{~km} / \mathrm{sec}$ during this period. The degree of satisfaction of Eq. (21) improves monotonically as the deformation progresses. Better satisfaction of Eq. (21) during the peak strain rate could be achieved by utilizing a smaller computational cell.

\subsection{Simulation duration}

The molecular dynamics simulation duration is another factor that warrants some consideration. Ideally, the simulation duration can be made much longer than the timescales for all chemical reactions and phase transitions that occur to ensure that the true end state of the shock is achieved. However, it is not possible in general to determine when the absolute final thermodynamic state of the simulation has been achieved without knowing some details about the system, and this method requires no prior knowledge of these details. Furthermore, maximum simulation times for molecular dynamics are typically on the nanosecond timescale for classical interatomic potentials and much shorter timescales for quantum approaches. The 
timescales for chemical reactions and phase transitions is much longer for many materials of interest. For example, chemistry in a detonating explosive can occur for microseconds or longer behind the shock front. For these reasons, it is necessary to perform all the simulations on the same timescale when calculating points on a shock Hugoniot using the technique presented here.

It might be expected that the Hugoniots calculated with this method and experimental measurements made on the same timescale would be in agreement. This timescale correspondence is a very loose criterion and the quality of agreement between simulations and experiments on timescales before the final thermodynamic state is reached likely depends on details of the particular material system. Some qualitative agreement between simulations and experiments on intermediate timescales is demonstrated for silicon in a later section of this chapter. Simulation timescale issues are discussed further in the following sections on double shock waves.

\section{TREATMENT OF MULTIPLE SHOCK WAVES}

The sections above describe the simulation of a single stable shock wave. However, it is not always possible for a single shock to take the molecular dynamics system to some pressures or particle velocities. For example, Figure 2 shows how it may not be possible to connect a straight Rayleigh line to all final pressures when there is a region of negative curvature in the Hugoniot, $\left.\frac{\partial^{2} p}{\partial v^{2}}\right|_{\text {Hugoniot }}<0$. Such regions of negative curvature are common in condensed phase materials and may be a result of phase transformations or may be the shape of a single phase Hugoniot. In Figure 2, it is not possible to connect state A to any state between $\mathrm{B}$ and $\mathrm{D}$ with a single straight Rayleigh line. Therefore it is not possible for a single shock wave to compress the system to a pressure between the pressures of states B and D. However, state B is a special state where the Rayleigh line from A to B is tangent to the Hugoniot implying a condition of neutral shock stability there, i.e. $u_{\mathrm{B}}+c_{\mathrm{B}}=\mathrm{v}_{\mathrm{s}}$. Therefore the mechanical stability condition for the first shock wave breaks down at state $\mathrm{B}$ and a second shock wave with a different speed can form. In the case of Figure 2, two Rayleigh lines are sufficient to shock the material to a pressure between that of states B and D. The first Rayleigh line goes from A to B and a second forms from B to C. The mechanical stability criteria are satisfied at points A and C.

The presence of places in the shock Hugoniot where $u+c=\mathrm{v}_{\mathrm{s}}$ can be detected using the method presented in this chapter without any prior knowledge of the shock Hugoniot. Figure 7 illustrates this process. If a plot of the final pressure (or particle velocity or volume) as a function of shock speed for multiple single wave simulations at various shock speeds is discontinuous in some pressure region, a state exists on the shock Hugoniot where $u+c=\mathrm{v}_{\mathrm{s}}$ and a second shock wave can form. This result can be seen by a geometrical argument based on the schematic Hugoniot in Figure 2. In Figure 7, states B and D correspond to those shown in Figure 2. The first wave shock speed for double wave simulations is chosen to be the smallest shock speed that takes the material to state D. This choice ensures that the simulation will progress beyond state B. We take the thermodynamic state of transition 
between the first and second waves to be the state where the thermodynamic variables change most slowly. Such a region is illustrated in Figure 8.

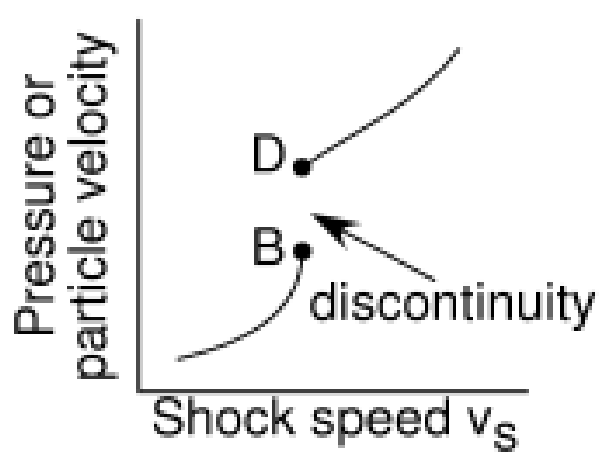

Figure 7. Schematic illustrating how regions on the Hugoniot where $u+c=\mathrm{v}_{\mathrm{s}}$ (which lead to the formation of a second shock wave) can be detected. If a plot of the final pressure (or particle velocity or volume) as a function of shock speed for multiple single wave simulations at various shock speeds is discontinuous in some pressure region, a state exists on the shock Hugoniot where $u+c=\mathrm{v}_{\mathrm{s}}$ and a second shock wave can form. States B and D in this plot correspond to those shown in Figure 2. The first wave shock speed for double wave simulations is chosen to the smallest shock speed that takes the material to state D.

Figure 8 shows the volume as a function of time for four overdriven single shock wave simulations in the [110] direction of a 25688 atom perfect Lennard-Jones face centered cubic crystal. Elastic compression is characterized by $V / V_{0} \sim 0.9$ and plastic compression occurs for smaller volumes. As the shock speed decreases, the amount of time the molecular dynamics system spends in the elastically compressed state increases. This plot illustrates how the final thermodynamic state in the shock is a function of the simulation duration when slow chemical reactions or phase transitions occur. For example, on the 10-20 ps timescale, the $2.8 \mathrm{~km} / \mathrm{sec}$ shock has an elastically compressed final state; on the $100 \mathrm{ps}$ timescale, this simulation has a plastically compressed final state.

The choice of a particular simulation timescale enables determination of the velocity of the first shock wave and the thermodynamic state where the transition between the first and second waves occurs. For example, simulations performed for 60 ps show plastic deformation for a $2.815 \mathrm{~km} / \mathrm{sec}$ shock speed but no plastic deformation for $2.8 \mathrm{~km} / \mathrm{sec}$. Therefore the first (elastic) wave speed for a double wave simulation is $2.815 \mathrm{~km} / \mathrm{sec}$ and the thermodynamic state at the transition between the two waves is the state where the slowest volume change occurs in the elastically compressed portion of the $2.815 \mathrm{~km} / \mathrm{sec}$ simulation. (These choices were utilized to produce Figure 9, to be discussed later.) The dependence of the Hugoniot elastic limit on the simulation time is discussed in more detail in the next section.

Each of the single wave simulations performed to construct a plot like in Figure 7 has physical validity regardless of the presence or lack of regions on the Hugoniot where a double shock wave can form. For this reason, it is possible to perform a physically valid single shock wave simulation without any knowledge of the existence of double shock waves. This 
property is particularly useful when computationally expensive molecular dynamics methods like tight-binding are utilized where calculation of the entire shock Hugoniot can be prohibitively expensive.

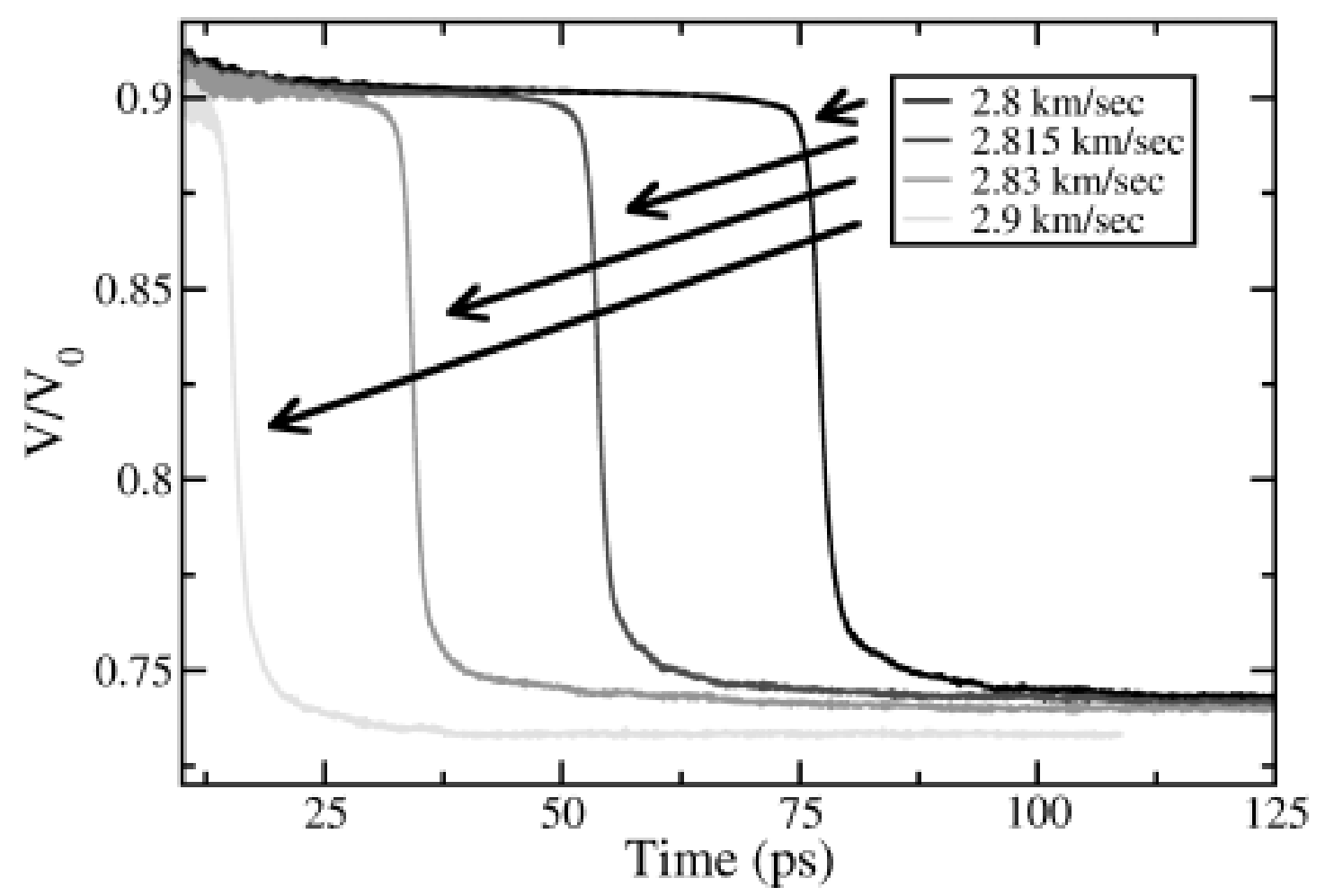

Figure 8. Volume as a function of time for four overdriven single shock wave simulations in the [110] direction of a 25688 atom perfect Lennard-Jones face centered cubic crystal. Elastic compression is characterized by $V / V_{0} \sim 0.9$ and plastic compression occurs for smaller volumes. As the shock speed decreases, the amount of time the molecular dynamics system spends in the elastically compressed state increases. This plot illustrates how the final thermodynamic state in the shock is a function of the simulation duration when slow chemical reactions or phase transitions occur. For example, on the 10$20 \mathrm{ps}$ timescale, the $2.8 \mathrm{~km} / \mathrm{sec}$ has an elastically compressed final state; on the $100 \mathrm{ps}$ timescale, this simulation has a plastically compressed final state.

The Hamiltonian Eq. (9) can be modified to constrain the molecular dynamics simulation to two or more Rayleigh lines. In the case of two lines, we utilize the form,

$$
\begin{aligned}
& H=\sum_{i, \alpha} \frac{P_{i, \alpha}^{2}}{2 m_{i} a_{\alpha}^{2}}+\phi\left(\left\{A \vec{s}_{i}\right\}\right)+\frac{P_{a_{x}}^{2}}{2 Q} \frac{M}{\left(a_{y} a_{z}\right)^{2}}-\theta\left(a_{x}-a_{x, 1}\right)\left[\frac{1}{2} M \mathrm{v}_{\mathrm{s}, 0}^{2}\left(1-\frac{a_{x}}{a_{x, 0}}\right)^{2}+p_{0} a_{y} a_{z}\left(a_{x, 0}-a_{x}\right)\right] \\
& -\theta\left(a_{x, 1}-a_{x}\right)\left[\frac{1}{2} M\left(u_{1}-\mathrm{v}_{\mathrm{s}, 1}\right)^{2}\left(1-\frac{a_{x}}{a_{x, 1}}\right)^{2}-p_{1} a_{y} a_{z}\left(a_{x, 1}-a_{x}\right)+\frac{1}{2} M \mathrm{v}_{\mathrm{s}, 0}^{2}\left(1-\frac{a_{x, 1}}{a_{x, 0}}\right)^{2}+p_{0} a_{y} a_{z}\left(a_{x, 0}-a_{x, 1}\right)\right]
\end{aligned}
$$


where $\theta(x)$ is the Heaviside function, $\mathrm{v}_{\mathrm{s}, 0}$ and $\mathrm{v}_{\mathrm{s}, 1}$ are the first and second wave shock speeds, respectively, and the quantities with subscript 1 are taken at the point of transition between the first and second waves. Specifically,

$u_{1}=\mathrm{v}_{\mathrm{s}, 0}\left(1-\frac{\rho_{0}}{\rho_{1}}\right)$

and

$p_{1}=p_{0}+\mathrm{v}_{\mathrm{s}, 0}^{2} \rho_{0}\left(1-\frac{\rho_{0}}{\rho_{1}}\right)$.

The equation of motion for the volume coordinate is,

$$
\begin{aligned}
& \dot{P}_{a_{x}}=\ddot{a}_{x} \frac{Q}{M}\left(a_{y} a_{z}\right)^{2}=\theta\left(a_{x}-a_{x, 1}\right)\left[a_{y} a_{z}\left(p-p_{0}\right)-\frac{M \mathrm{v}_{\mathrm{s}, 0}^{2}}{a_{x, 0}}\left(1-\frac{a_{x}}{a_{x, 0}}\right)\right] \\
& +\theta\left(a_{x, 1}-a_{x}\right)\left[a_{y} a_{z}\left(p-p_{1}\right)-\frac{M\left(\mathrm{v}_{\mathrm{s}, 1}-u_{1}\right)^{2}}{a_{x, 1}}\left(1-\frac{a_{x}}{a_{x, 1}}\right)\right]
\end{aligned}
$$

The extension to three or more waves can be accomplished in a similar fashion.

\subsection{Time-dependence of the $p-v$ space path}

The formation and evolution of multiple waves becomes more complicated when chemical reactions or phase transitions occur. Volume decreasing phase transformations cause the pressure at point B in Figure 2 and Figure 7 to decrease with time. This common phenomenon is known as elastic precursor decay in elastic-plastic wave system. [9] The timescale for this pressure decay depends primarily on the timescale for the chemical reaction or phase transition that gives rise to the $2^{\text {nd }}$ wave.

In a double shock wave with chemical reactions, unsteady behavior can lead to a $p$ - $v$ space path that is not necessarily well described by Rayleigh lines. However, we assume here that for a given period of time the $p-v$ space path can be transiently approximated by a set of Rayleigh lines. This description is valid when the timescale of the pressure change at point $\mathrm{B}$ in Figure 2 is less than the time required for a material element to progress from the initial state to the final shocked state. A more quantitative version of this statement is formulated in the remainder of this section.

For the simulations performed using the method described in this chapter, the rate at which the pressure at point B (denote this pressure $p_{1}$ ) decreases can be determined using the socalled shock change equation. $[15,16]$ For purposes here, we assume the internal energy can now be expressed as $e=e(p(x, t), v(x, t), \lambda(x, t))$ where $\lambda$ is a generalized reaction parameter for a reaction or phase transition, $0 \leq \lambda \leq 1$. The rate of pressure change in the moving frame of the shock wave at the metastable point B can be obtained from the so-called shock change equation, 
$\frac{d p_{1}}{d t}=\frac{\rho_{1} c_{1}^{2}\left[\sigma \dot{\lambda}-\frac{\partial p_{1}}{\partial x} \frac{\eta}{\rho_{0} \mathrm{v}_{\mathrm{s}, 0}}\right]}{1+\left.\rho_{0} \mathrm{v}_{\mathrm{s}, 0}(1-\eta)^{-1} \frac{\partial u_{1}}{\partial p}\right|_{\text {Hugoniot }}}$

where $\eta \equiv 1-\frac{\left(u_{1}-\mathrm{v}_{\mathrm{s}, 0}\right)^{2}}{c_{1}^{2}}, c_{1}$ is the local longitudinal sound speed, $\left.\sigma \equiv \rho_{1} \frac{\partial \tilde{v}_{1}}{\partial \lambda}\right|_{S, p}$ where the derivative is taken at constant pressure along an isentrope, $\mathrm{v}_{\mathrm{s}, 0}$ is the speed of the first shock wave of the pair, and all variables with subscript 1 are taken to be at the transition point between the first and second waves (state B in Figure 2). Equation (23) can be obtained by starting with Equations (1), (2), and (3) and calculating the pressure at a point moving at the shock speed, i.e. $\frac{d p_{1}}{d t}=\frac{\partial p}{\partial t}+\mathrm{v}_{\mathrm{s}} \frac{\partial p}{\partial x}$. A complete derivation can be found in Ref. [9].

Eq. (23) can be simplified considerably in the case of interest here. The stability condition at the transition state between the two waves is $u_{1}+c_{1}=\mathrm{v}_{\mathrm{s}}$ which leads to the result that $\eta=0$ at that point. Furthermore the fact that the Rayleigh line for the first shock and the Hugoniot share a common tangent at the transition state of interest (giving rise to the condition $u_{1}+c_{1}=\mathrm{v}_{\mathrm{s}}$ ) leads the result,

$\left.\frac{\partial u_{1}}{\partial p}\right|_{\text {Hugoniot }}=\left.\frac{\partial u}{\partial p}\right|_{\text {Rayleigh line }}=\frac{1}{\rho_{0} \mathrm{v}_{\mathrm{s}, 0}}$

in this case, taking $u_{0}=0$. These simplifications lead the to the result,

$\frac{d p_{1}}{d t}=\frac{\rho_{1}\left(\mathrm{v}_{\mathrm{s}, 0}-u_{1}\right)^{2} \sigma \dot{\lambda}}{2}$

Unfortunately, the parameter $\left.\sigma \dot{\lambda} \equiv \rho_{1} \dot{\lambda} \frac{\partial \tilde{v}_{1}}{\partial \lambda}\right|_{S, p}$ cannot be determined directly from information obtained form a simulation using the method presented in this chapter since the stress condition of the material lies along a Rayleigh line, not constant stress. However, we estimate

$$
\left.\sigma \dot{\lambda} \approx \rho_{1} \frac{\Delta \tilde{v}}{\Delta t}\right|_{\min }
$$

where $\left.\frac{\Delta \tilde{v}}{\Delta t}\right|_{\min }$ is the minimum rate of change in specific volume during the simulation. The minimum rate occurs just after the transition to the $2^{\text {nd }}$ wave occurs, i.e. from 0.5 to 2 picoseconds in Figure 4, or state B in Figure 2. We expect that Eq. (25) provides an upper bound on the actual value of $\sigma \dot{\lambda}$ if $\dot{\lambda}$ has the form $\dot{\lambda} \sim\left(p_{1}-p^{\prime}\right)$ since we calculate this parameter along a Rayleigh line rather than a constant stress. With these simplifications, Eq. (23) becomes,

$$
\left.\frac{d p_{1}}{d t} \approx \frac{1}{2} \frac{\Delta \tilde{v}}{\Delta t}\right|_{\min } \rho_{1}^{2}\left(\mathrm{v}_{\mathrm{s}, 0}-u_{1}\right)^{2}
$$


This approximate form of the shock change equation enables the estimation of pressure decay of the first wave using information that can be obtained directly from the simulations. The approximation of the $p-v$ space path by more than one Rayleigh line in the case of volume decreasing reactions is justified when the Rayleigh lines do not change appreciably during the simulation, i.e.

$$
\left.\frac{d p_{1}}{d t} \approx \frac{1}{2} \frac{\Delta \tilde{v}}{\Delta t}\right|_{\min } \rho_{1}^{2}\left(\mathrm{v}_{\mathrm{s}, 0}-u_{1}\right)^{2}<<-\frac{\Delta p}{\Delta t}
$$

where $\Delta p$ and $\Delta t$ are chosen to be the pressure change of the second shock wave and time duration of a given simulation respectively. Any overestimation of reaction rates through the use of $\left.\frac{\Delta \tilde{v}}{\Delta t}\right|_{\min }$ makes Eq. (27) more stringent. The rough and approximate criterion provided by Eq. (27) can be used to assess the validity of a two-wave simulation. All of the parameters in Eq. (27) can be determined from a two-wave simulation after it has completed. Alternatively, Eq. (27) provides a relation for the maximum duration of a simulation $\Delta t$ can be performed without appreciable change in the $p-v$ space through which the shock takes the material. This relation may be useful when multiple chemical reactions or phase transitions of disparate time scales exist, where a fast reaction gives rise to a large value of $\frac{d p_{1}}{d t}$ but slower reactions exist that prevent a final state from being reached before the Rayleigh line validity condition Eq. (27) breaks down. It is not necessary to have prior knowledge of the number, type, or any other details of chemical reactions or phase transitions to utilize the techniques presented in this chapter.

The Rayleigh line validity condition Eq. (27) can be shown to be valid for long wave propagation times. By considering a reaction rate of the form $\dot{\lambda}=\alpha\left(p_{1}-p^{\prime}\right)$, the shock change equation, Eq. (24) gives,

$$
p_{1}=\left(p_{1}^{0}-p^{\prime}\right) \exp \left(\frac{\rho_{1}\left(\mathrm{v}_{\mathrm{s}, 0}-u_{1}\right)^{2} \sigma \alpha}{2} t\right)+p^{\prime}
$$

where $p_{1}^{0}$ is the initial pressure of the first shock wave. Note that $\alpha>0$ and $\sigma<0$ here. In a shock wave, the time between the arrival of the first shock and the final pressure of the second shock is attained ( $\Delta t$ in Eq. (27)) has an upper bound that is determined by the speed of the two shock waves and the particle velocity between them. It can be easily shown that $\Delta t$ in this upper bound case scales linearly with the time that the shock waves have been propagating. The exponential time-dependence of $p_{1}$ and the linear time-dependence of $\Delta t$ imply Eq. (27) is always satisfied after the shock has propagated for some period of time. During times when this condition is not satisfied, the $p-v$ space path a material element follows is more complicated than straight Rayleigh lines, but such situations are transient. Therefore it is expected that the approximation of the $p-v$ space path with a series of Rayleigh lines is valid for shock waves that have propagated for some period of time in most systems.

In practice, we find that the Rayleigh line validity condition Eq. (27) holds when the lifetime of the elastically strained state is appreciable, as it is for the slower shock speed 
simulations in Figure 8. It breaks down for simulations where relatively little time is spent in the elastically compressed state before plastic deformation occurs.

\section{APPLICATION TO A LENNARD-JONES CRYSTAL}

Figure 9 presents the calculated Hugoniot for shock waves propagating in the [110] direction of a Lennard-Jones face-centered cubic crystal of 25688 atoms. All results that follow are given in LJ units of $\sigma, \varepsilon$ and $m$. The end states were taken around $\mathrm{t}=60$. The integration time step was $1.15 \times 10^{-4}$, and volume mass-like parameter $Q=2.737 \times 10^{-3}$. $v_{0}=0.9617, T_{0}=0.01$. Longitudinal sound speed in the [110] direction $c_{0}=9.5$. For the double-wave simulations, the Hugoniot elastic limit (HEL) volume and shock speed were determined to be $v_{1} / v_{0}=0.9011$ and $\mathrm{v}_{\mathrm{S}} / c_{0}=1.818$. These are the volume of the transition between first and second waves and shock speed of the first wave, respectively. A choice of $Q$ sufficient to ensure only elastic deformation occurs during the initial volume oscillations was verified by monitoring the radial distribution function during elastic oscillations during one of these simulations.

The black triangles in Figure 9 show results of single wave simulations. These simulations show that a gap exists between about $v / v_{0}=0.74$ and $v / v_{0}=0.9$. As in Figure 7, this gap indicates the existence of a shock instability leading to the formation of a second wave. Figure 9 shows good agreement with NEMD volume data in the double shock regime.

Figure 9 shows quantitative temperature agreement with NEMD for single wave simulations and double wave simulations with high plastic wave speeds $\left(v / v_{0}>0.76\right)$, where we find qualitative agreement. Further study of both NEMD convergence (timescale issues), which is affected by slow plastic relaxation, and multiscale methods in this regime is desirable. In addition to timescale issues, a possible origin of the temperature difference is the difference in calculated HEL. NEMD simulations show a HEL volume of $v_{1} / v_{0} \approx 0.91$, [17] which is slightly greater than the HEL for the multiscale simulations $\left(v_{1} / v_{0}=0.9011\right)$. This difference is consistent with the observation of a higher temperature.

\section{APPLICATION TO CRYSTALLINE SILICON}

Crystalline silicon is another material that exhibits shock wave splitting due to phase transitions. Figure 10 shows shock speed as a function of particle velocity for shock waves propagating in the [110] direction in silicon described by the Stillinger-Weber potential. [18] This potential has been found to provide a qualitative representation of some condensed properties of silicon. Data calculated using the NEMD method are compared with results of the method presented in this chapter. NEMD simulations were done with a computational cell of size $920 \AA x 12 \AA x 11 \AA$ (5760 atoms) for a duration of about 10-20ps. Simulations with the multiscale method were done with a computational cell size of $19 \AA \AA x 12 \AA x \times 11 \AA$ (120 atoms.) Both simulations were started at $300 \mathrm{~K}$ and zero stress. Since the NEMD simulations were limited to the $10 \mathrm{ps}$ timescale, simulations with the multiscale method were performed to calculate the Hugoniot on this 10ps timescale for comparison. The final particle velocity in these simulations was taken to be a point of steady state after a few ps. 


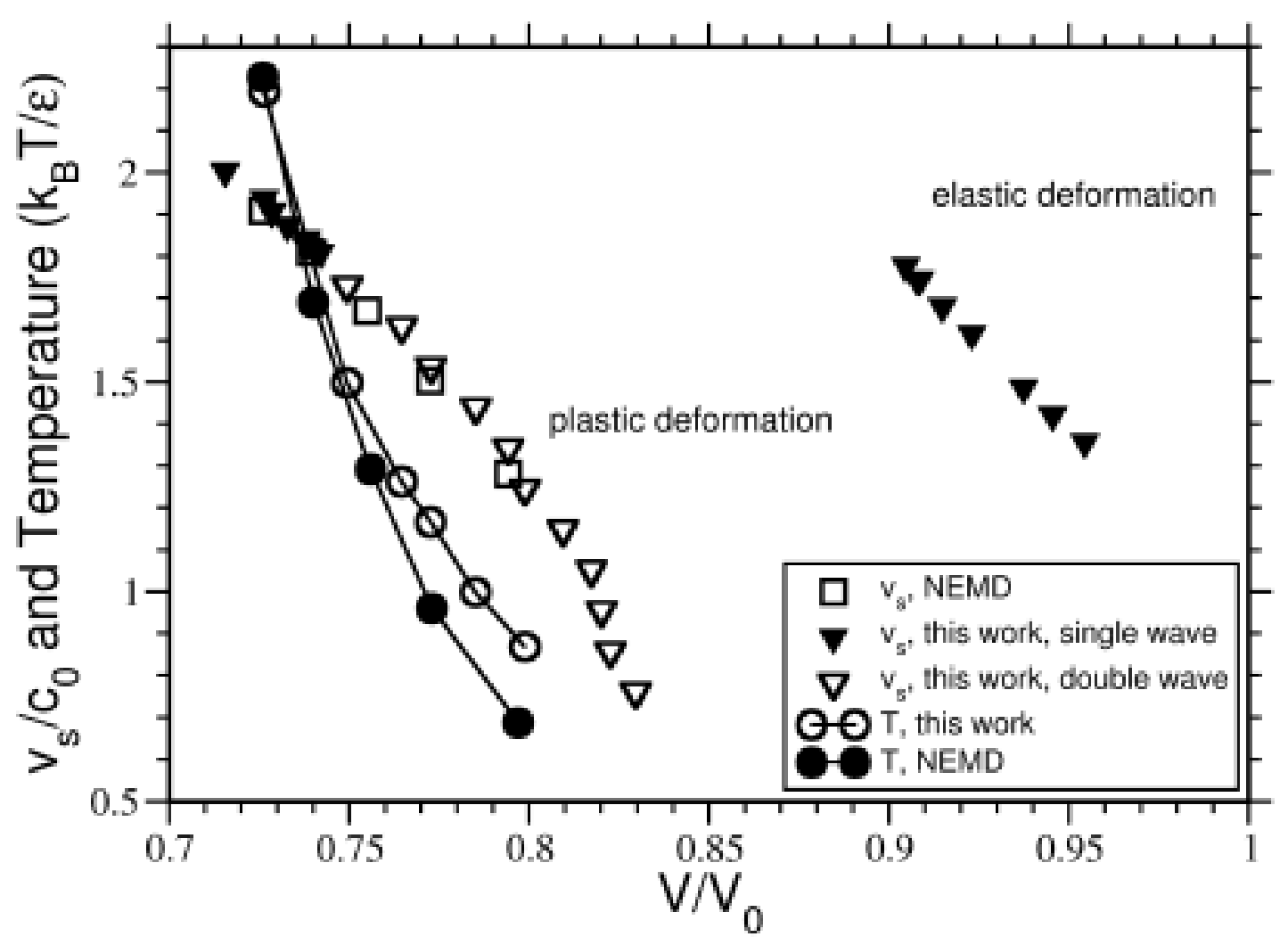

Figure 9: Calculated Hugoniot for shocks in the [110] direction of perfect 25688 atom Lennard-Jones face-centered cubic crystal. The NEMD shock speed and temperature data are from Ref.13. Here, $c_{0}=9.5$ in Lennard Jones units. See text for details.

Figure 10 indicates a single shock wave exists below $1.9 \mathrm{~km} / \mathrm{sec}$ particle velocity. Above this particle velocity, the elastic shock wave precedes a slower moving shock characterized by plastic deformation. Agreement between the two methods is good for all regions except for the plastic wave speed for particle velocities less than $2.1 \mathrm{~km} / \mathrm{sec}$. The wide range of values for the plastic wave speeds in NEMD simulations in this regime is likely due to finite simulation cell size effects which are not present in the simulations shown in Figure 9 for Lennard-Jones.

The Rayleigh line validity condition Eq. (27) is satisfied for the simulations performed in the two-shock regime, giving a typical value for $-\frac{d p_{1}}{d t}$ of $0.1 \mathrm{GPa} / \mathrm{ps}$, while $\frac{\Delta p}{\Delta t}$ is greater than $0.5 \mathrm{GPa} / \mathrm{ps}$ for all simulations in Figure 10 .

One of the primary advantages of using the method outlined in this chapter is the ability to simulate for much longer times than is possible with NEMD. As an example, Figure 10 shows the result of a $5 \mathrm{~ns}$ simulation performed along a Rayleigh line corresponding to a shock speed of $10.3 \mathrm{~km} / \mathrm{sec}$. The uniaxially compressed elastic state required $5 \mathrm{~ns}$ to undergo plastic deformation. The difference in particle velocity between the 10 ps and 5 ns simulations at this shock speed is $0.8 \mathrm{~km} / \mathrm{sec}$, suggesting that the elastically compressed state 
is metastable with an anomalously large lifetime. While some caution should be taken when attributing physical significance to this result from the empirical potential of Stillinger and Weber, this result is qualitatively consistent with experimental observations of shocked silicon that indicate an anomalously high pressure elastic wave exists on the nanosecond timescale. [4]

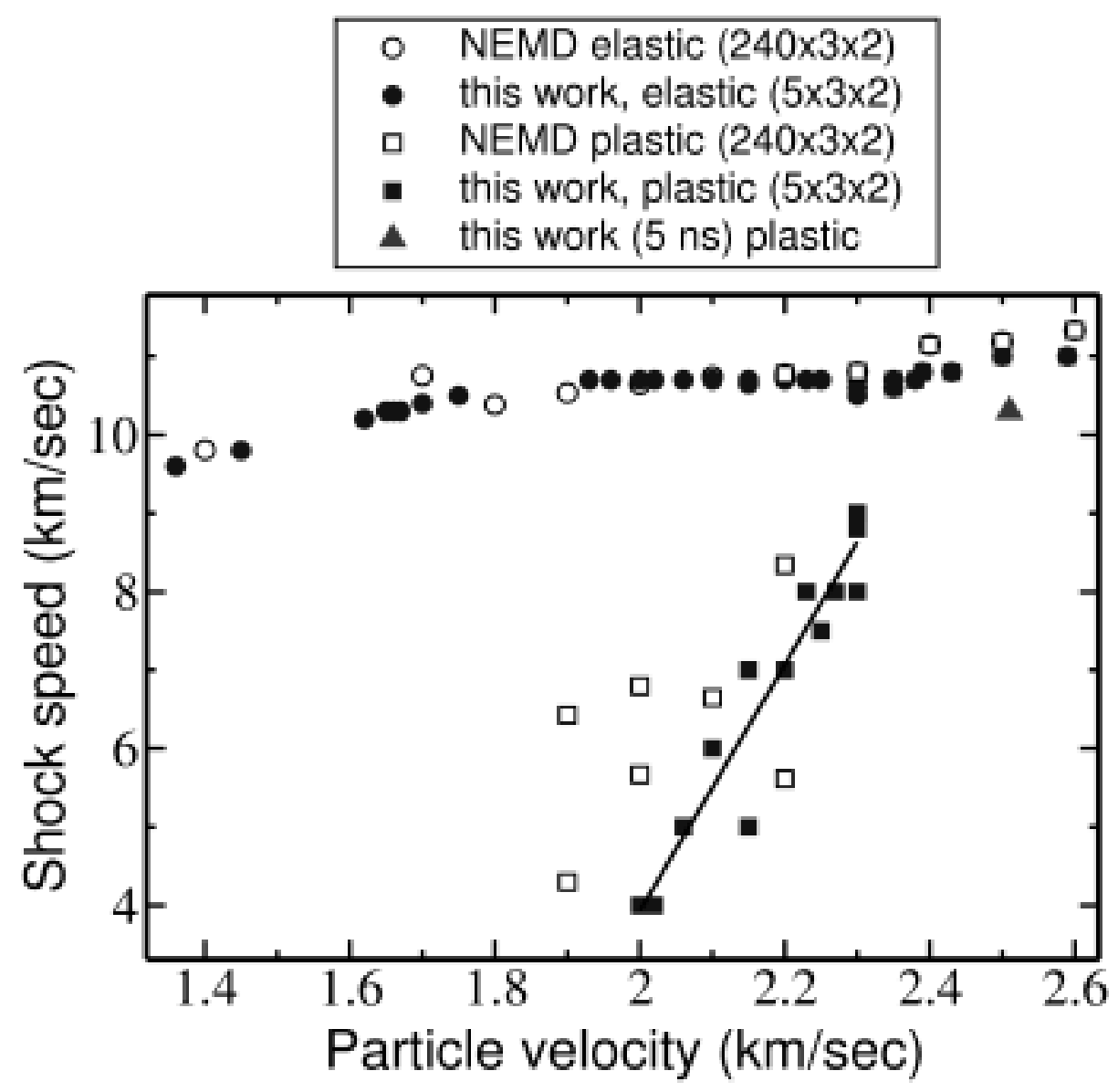

Figure 10. Hugoniot for shocks in the [110] direction of a 5760 atom Stillinger-Weber silicon perfect diamond structure crystal. The black line is an aid to the eye. The end state for all simulations was taken on the 10 picosecond timescale except for the red triangle data point which was taken after 5 nanoseconds.

The 5 ns simulation demonstrated a substantial computational savings over the NEMD method. For an $O(N)$ method of force evaluation, the computational cost of this simulation with the NEMD method would be at least $10^{5}$ times greater than the multiscale method.

\section{APPLICATION TO NITROMETHANE}

Chemistry in detonating explosives can occur long after the shock front has passed and an accurate description of the chemical reactions in these materials generally requires use of a tight-binding or more accurate molecular dynamics approach. The multiscale method presented in this chapter has the biggest advantages over NEMD and other methods for simulations of long duration with computationally expensive molecular dynamics methods. 
Furthermore, most practical energetic materials are molecular solids that have relatively short atomic correlation lengths under detonation conditions. This enables the use of small simulation cells and satisfaction of the strain rate condition Eq. (21) shortly behind the shock front.

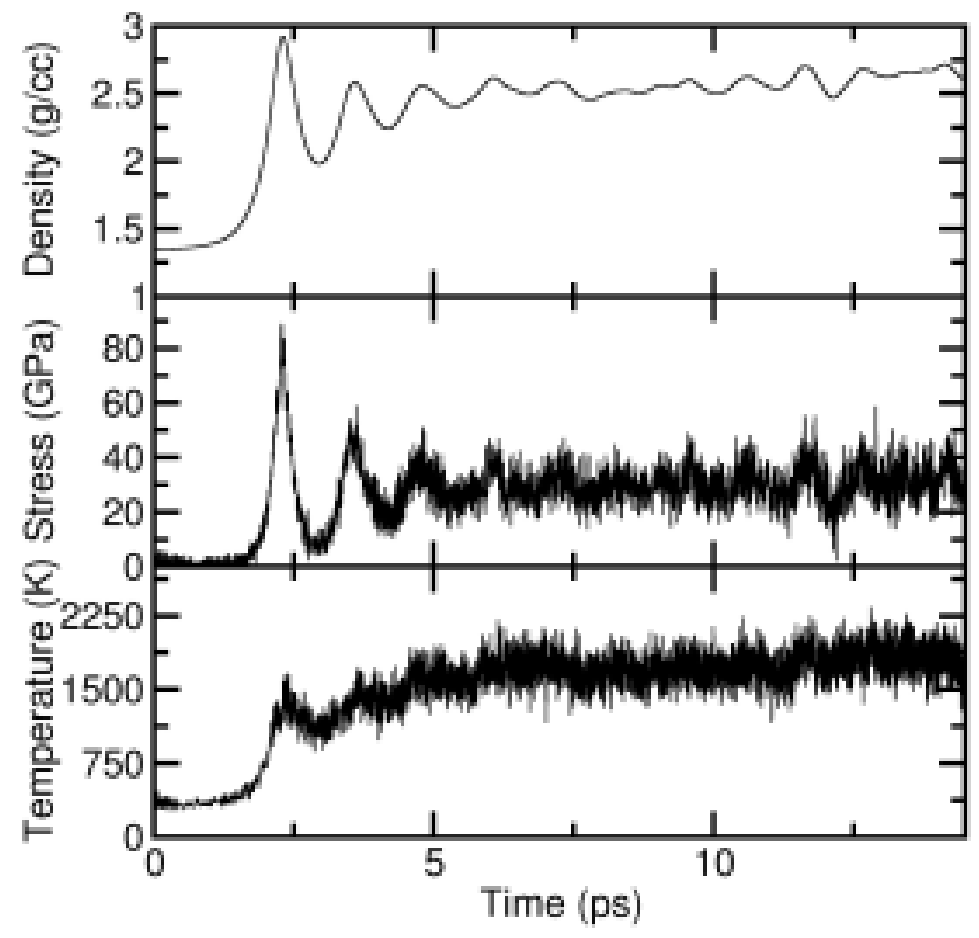

Figure 11. Time-dependence of density, uniaxial stress, and temperature for a $7 \mathrm{~km} / \mathrm{sec}$ shock in nitromethane.

As an example study case, we have applied the multiscale method to nitromethane, experiencing shock compression along the c axis (longest axis of the primitive cell) with a shock speed of $7 \mathrm{~km} / \mathrm{sec}$. The initial density is $1.34 \mathrm{~g} / \mathrm{cc}$, initial temperature is around $300 \mathrm{~K}$, and initial stress is around $0.5 \mathrm{GPa}$. The atomic energies and forces were computed using the SCC-DFTB method, [19] utilizing a supercell of solid crystalline nitromethane containing eight molecules (56 atoms). The supercell was obtained by doubling the primitive cell in the c lattice direction. The SCC-DFTB method is an extension of the standard tight binding approach [20] within the context of density-functional theory [21] and provides a selfconsistent description of total energies, atomic forces, and charge transfer. The dynamics were followed up to $17.5 \mathrm{ps}$ with an integration time step of $0.2 \mathrm{fs}$. Performing this simulation with the NEMD method would require $10^{5}$ to $10^{6}$ times more computational effort due to the roughly $O\left(N^{3}\right)$ scaling of the computational work with number of atoms. 
Figure 11 shows the time profile of the density, stress, and temperature of the system throughout the simulation. Density oscillations are damped within a few oscillations. Examination of the simulation cell contents shows that at $4.9 \mathrm{ps}$, a proton transfer process occurs, which can be described as: $\mathrm{CH}_{3} \mathrm{NO}_{2}+\mathrm{CH}_{3} \mathrm{NO}_{2} \rightarrow \mathrm{CH}_{3} \mathrm{NO}_{2} \mathrm{H}+\mathrm{CH}_{2} \mathrm{NO}_{2} \rightarrow \mathrm{CH}_{3} \mathrm{NO}_{2}$ $+\mathrm{CH}_{2} \mathrm{NO}_{2} \mathrm{H}$. This chemical event that leads to the formation of the so-called aci acid $\mathrm{H}_{2} \mathrm{CNO}_{2} \mathrm{H}$ moiety persists for over 4 ps of the simulation. There have been several experimental concurrences for the production of the aci ion in highly pressurized and detonating nitromethane. Shaw et al. [22] observed that the time to explosion for deuterated nitromethane is about ten times longer than that for the protonated materials, suggesting that a proton (or hydrogen atom) abstraction is the rate-determining step. Isotope-exchange experiments provided evidence that the aci ion concentration is increased upon increasing pressure, [23] and UV sensitization of nitromethane to detonation was shown to correlate with the aci ion presence. [24]

\section{CONCLUSION}

In this chapter we have presented a multi-scale method for molecular dynamics simulations of shock compression and characterized its behaviour. This method attempts to constrain the molecular dynamics system to the sequence of thermodynamic states that occur in a shock wave. While we have presented one particular approach, it is certainly not unique and there are likely a variety of related approaches to multi-scale simulations that have a variety of differing practical properties. These methods open the door to simulations of shock propagation on the longest timescales accessible by molecular dynamics and the use of accurate but computationally costly material descriptions like density functional theory. It is our belief that this method promises to be a valuable tool for elucidation of new science in shocked condensed matter.

\section{REFERENCES}

[1] C. S. Yoo, W. J. Nellis, M. L. Sattler, and R. G. Musket, Appl. Phys. Lett., 61 (1992) 273.

[2] W. J. Nellis, S. T. Weir, and A. C. Mitchell, Science, 273 (1996) 936; S. T. Weir, A. C. Mitchell, and W. J. Nellis, Phys. Rev. Lett., 76 (1996) 1860.

[3] M. D. Knudson and Y. M. Gupta, Phys. Rev. Lett., 81 (1998) 2938.

[4] Loveridge-Smith, A. Allen, J. Belak, T. Boehly, A. Hauer, B. Holian, D. Kalantar, G. Kyrala, R. W. Lee, P. Lohmdahl, M. A. Meyers, D. Paisley, S. Pollaine, B. Remington, D. C. Swift, S. Weber and J. S. Wark, Phys. Rev. Lett., 86 (2001) 2349.

[5] K. Kadau, T. C. Germann, P. S. Lomdahl, and B. L. Holian, Science 296, 1681 (2002); J. D. Kress, S. R. Bickham, L. A. Collins, B. L. Holian, and S. Goedecker, Phys. Rev. Lett., 83 (1999) 3896.

[6] E. J. Reed, L. E. Fried, and J. D. Joannopoulos, Phys. Rev. Lett., 90 (2003) 235503.

[7] M. P. Allen and D. J. Tildesley, Computer simulation of liquids (Oxford University Press, New York, 1989).

[8] H. Goldstein, Classical Mechanics (Addison-Wesley, Reading, MA, 1980).

[9] G. E. Duvall in Proceedings of the International School of Physics, Physics of High Energy Density (Academic Press, New York, 1971).

[10] Y. B. Zel'dovich and Y.P.Raizer, Physics of shock waves and high-temperature hydrodynamic phenomena (Academic Press, New York, NY, 1967). 
[11] H. C. Anderson, J. Chem. Phys., 72 (1980) 2384.

[12] J. Fox and H. C. Andersen, J. Chem. Phys., 88 (1984) 4019.

[13] R. Ravelo, B. L. Holian, T. C. Germann, private communication.

[14] B. L. Holian, A. F. Voter, N. J. Wagner, R. J. Ravelo, S. P. Chen, W. G. Hoover, C. G. Hoover, J. E. Hammerberg and T. D. Dontje, Phys. Rev. A, 43 (1991) 2655.

[15] E. Jouget, Mecanique des explosifs (Octave Doin et Fils, Paris, 1917).

[16] W. Fickett and W. Davis, Detonation, (University of Califronia Press, Berkeley, CA, 1979).

[17] T. C. Germann, B. L. Holian, P. S. Lomdahl, and R. Ravelo, Phys. Rev. Lett., 84 (2000) 5351.

[18] F. H. Stillinger and T. A. Weber, Phys. Rev. B, 31 (1985) 5262.

[19] M. Elstner, D. Porezag, G. Jungnickel, J. Elsner, M. Hauk, T. Frauenheim, S. Suhai, and G. Seifert, Phys. Rev. B, 58 (1998) 7260.

[20] J. C. Slater and G. F. Koster, Phys. Rev., 94 (1954) 1498.

[21] P. Hohenberg and W. Kohn, Phys. Rev., 136 (1964) B864.

[22] R. Shaw, P. S. Decarli, D. S. Ross, E. L. Lee, and H. D. Stromberg, Combust. Flame, 50 (1983) 123; R. Shaw, P. S. Decarli, D. S. Ross, E. L. Lee, and H. D. Stromberg, Combust. Flame, 35 (1979) 237.

[23] R. Engelke, D. Schiferl, C. B. Storm, and W. L. Earl, J. Phys. Chem., 92 (1988) 6815.

[24] R. Engelke, W. L. Earl, and C. M. Rohlfing, J. Phys. Chem., 90 (1986) 545.

This work was performed under the auspices of the U.S. Department of Energy by University of California, Lawrence Livermore National Laboratory under contract W-7405-Eng-48. 